JOURNAL OF THE

AMERICAN MATHEMATICAL SOCIETY

Volume 25, Number 2, April 2012, Pages 555-583

S 0894-0347(2011)00724-8

Article electronically published on December 1, 2011

\title{
TOWARD THE FOURIER LAW FOR A WEAKLY INTERACTING ANHARMONIC CRYSTAL
}

\author{
CARLANGELO LIVERANI AND STEFANO OLLA
}

\section{Contents}

1. Introduction

2. The model 558

3. The results 559

4. Proof of main theorem 561

4.1. Tightness 561

4.2. Identification of the limit 562

5. Hypoellipticity and regularity on energy shells 566

6. Hypocoercivity 567

7. Global regularity 56

7.1. The transversal direction $\quad 569$

7.2. Transversal regularity 569

8. Structure and regularity of $\gamma^{2}$ and $\alpha \quad 571$

Appendix A. Commutators 573

Appendix B. Hypocoercivity estimates 574

Appendix C. A coordinate change 577

Appendix D. Microcanonical measure 578

Appendix E. The Gaussian case 581

Acknowledgments $\quad 583$

References 583

\section{INTRODUCTION}

The problem of deriving the heat equation and Fourier's law for the macroscopic evolution of the energy from a microscopic dynamics of interacting atoms (Hamiltonian or quantum), is one of the major goals of nonequilibrium statistical mechanics [2].

Although we are still very far from a rigorous mathematical derivation, we now have some understanding of the needed ingredients.

Received by the editors January 18, 2011 and, in revised form, November 6, 2011.

2010 Mathematics Subject Classification. Primary 82C70, 60F17, 80A20.

Key words and phrases. Weak coupling, scaling limits, hypoellipticity, hypocoercivity, Ginzburg-Landau dynamics, heat equation.

This paper has been partially supported by the European Advanced Grant Macroscopic Laws and Dynamical Systems (MALADY) (ERC AdG 246953), by Agence Nationale de la Recherche, under grant ANR-07-BLAN-2-184264 (LHMSHE), and by MIUR under the grant PRIN 2007B3RBEY.

(C)2011 American Mathematical Society Reverts to public domain 28 years from publication 
It is clear that the heat equation is a macroscopic phenomenon, emerging after a diffusive rescaling of space and time. It is also clear that nonlinearities of the microscopic dynamics are necessary, since in a linear system of interacting oscillators, energy may disperse ballistically and thermal conductivity is infinite [12]. Nonlinearities of the interaction should give enough chaoticity and time mixing such that locally the system, in the macroscopic time scale, is in a state of local equilibrium. This should be intended in terms of a scale parameter $\varepsilon$ : in a region of linear size $\varepsilon$, at a large time scale $\varepsilon^{-b} t$, the system should be close to equilibrium with temperature given by the local average of kinetic energy. This statement of local equilibrium should be intended in the stronger sense that locally the dynamics is close to an equilibrium dynamics. Since energy is a conserved quantity, it can only evolve by moving between different regions of linear size $\varepsilon^{-1}$ through energy currents. Because of the size of the regions and the fact that in equilibrium energy currents have null average, one should look at time of the order $\varepsilon^{-2} t$ in order to see some exchange of energy between boxes at different temperatures. In other words a central limit theorem for the energy currents is involved, and the thermal conductivity is then given by the space-time integral of the current-current correlation (Green-Kubo formula). This conductivity will be convergent if the system in equilibrium has enough mixing properties.

To perform the above program, in a mathematically rigorous way, from a purely deterministic Hamiltonian dynamics, it is at the moment a too difficult challenge.

In recent years some mathematical results have been obtained by perturbing the dynamics with energy-conserving stochastic forces. The purpose of these stochastic perturbations is to give the ergodic and chaotic properties to the system without modifying the macroscopic behavior of the evolution of the energy.

This strategy has proven successful for systems in the hyperbolic scaling $(b=1)$, when momentum conservation is also preserved by the stochastic perturbations [11, obtaining an Euler system of equations for a compressible gas as a macroscopic equation, at least in the smooth regime.

In the diffusive scaling $(b=2)$ this problem is still very challenging even in the presence of the stochastic perturbations. The difficulty is essentially involved in the space-time rescaling and the corresponding central limit theorem.

In this paper we develop a weak-coupling approach to the problem of energy diffusion that permits us to separate the time limit from the space one. We consider a finite system of anharmonic oscillators, whose Hamiltonian dynamics is perturbed by a noise that conserves the kinetic energy of each oscillator (we consider oscillators that have at least 2 degrees of freedom). The noise could be thought of as modeling some chaotic internal degree of freedom of each atom.

The oscillators are weakly coupled with a small parameter $\varepsilon$. Consequently the exchange of energy between oscillators is given by the currents associated to the Hamiltonian mechanism, but multiplied by $\varepsilon$. The noise drives each atom towards (microcanonical) equilibrium where currents have null average. In a time scale of order $\varepsilon^{-2}$ current fluctuations are able to move energy around the lattice if gradients of energy are present between atoms.

We prove in fact that in the limit as $\varepsilon \rightarrow 0$, in the time scale $\varepsilon^{-2} t$, the energies of the atoms evolve autonomously following the solution of a system of stochastic differential equations, conservative of the total energy (cf. (3.8)). It turns out that 
this macroscopic stochastic evolution has already been considered in the hydrodynamic limit literature, and it is called the nongradient Ginzburg-Landau model [14. Consequently, using the techniques developed in [14, one could try to prove that under space-time diffusive rescaling, the energy evolves following a nonlinear heat equation. The results in 14 do not apply directly to (3.8) due to degeneracy of the coefficients, but hopefully they can be adapted to the present situation; for the moment we postpone this problem. We have thus reduced the derivation of the heat equation to a two-step procedure of which this paper rigorously accomplishes the first step.

We should remark here that an extension of the nongradient technique of 14 directly to our original microscopic stochastic dynamics would be much more challenging, as this dynamics is very degenerate. A recent development in this direction is done in [10, with a less degenerate energy-conserving noise that acts also on the positions.

The reason for the name nongradient comes from the fact that the currents of the macroscopic dynamics are not gradient, i.e. are not given by the spatial gradient of a local function of the configurations of energies. It is interesting to note that in the purely harmonic case, the macroscopic dynamics (3.8) become gradient (see Appendix E). This implies a connection between the nongradient property and nonlinearity of the microscopic dynamics. We also notice that in the purely harmonic case, because of the presence of the energy-conserving noise, the microscopic energy current has an exact fluctuation-dissipation decomposition in a gradient plus a fast fluctuating term (see formula (E.1) in Appendix E), which has already been exploited in [1] to obtain Fourier's law.

The main strategy of the proof is similar to other averaging principles $([5,6,8])$ : at a large time scale the dynamics of each atom is close to the equilibrium dynamics parametrized by its own energy. Energies of the atoms are our slow variables and evolve through their currents. But a simple averaging of these currents (which would occur in a time scale of order $\varepsilon^{-1}$ ) would not move any energy, since currents have null averages with respect to all equilibrium measures (microcanonical). This forces us to look at the time scale $\varepsilon^{-2}$, when the energy evolves due to the fluctuations of the currents in equilibrium. Thus we must establish a central limit theorem for the energy currents in the uncoupled dynamics; i.e., we have to study the Poisson equation

$$
L_{0} u=j,
$$

where $L_{0}$ is the generator of the uncoupled dynamics, and $j$ is the energy current between two particles. In order to prove our theorem, we need existence and regularity of the solution $u$ of this equation. The generator $L_{0}$ turns out to be hypoelliptic on each microcanonical energy surface, which provides regularity on the tangent direction of this surface. Yet, as the energy is exchanged from one particle to the other, we also need to prove regularity in nontangential directions.

For the existence of $u$, we prove a spectral gap in a proper Sobolev space, with an adaptation of hypocoercivity techniques [15. These techniques provide a precise control of this spectral gap with respect to the energy, a control especially needed at low energies. Such detailed information is necessary in order to perform the closure of the macroscopic equations.

In a companion article [4, a similar weak coupling limit is taken, but the oneatom dynamics is given by a chaotic strictly hyperbolic deterministic dynamics. The 
limit evolution of the energies is given again by a conservative Ginzburg-Landau dynamics, but the coefficients have a different behavior for lower energies.

\section{THE MODEL}

Let us consider a region $\Lambda \subset \mathbb{Z}^{d}$, set $N=|\Lambda|$, the number of sites in $\Lambda$. At each site we have a $\nu$-dimensional, $\nu \geq 2$, nonlinear oscillator, and we assume that such oscillators interact weakly via a nonlinear potential. Such a situation is described by the following Hamiltonian in the variables $\left(q_{i}, p_{i}\right)_{i \in \Lambda} \in \mathbb{R}^{2 \nu N}$ :

$$
H_{\varepsilon}^{\Lambda}:=\sum_{i \in \Lambda} \frac{1}{2}\left\|p_{i}\right\|^{2}+\sum_{i \in \Lambda} U\left(q_{i}\right)+\varepsilon \frac{1}{2} \sum_{|i-j|=1} V\left(q_{i}-q_{j}\right),
$$

where $U, V \in \mathcal{C}^{\infty}\left(\mathbb{R}^{\nu}, \mathbb{R}\right)$. We use the convention $\sum_{|i-j|=1}=\sum_{i \in \Lambda} \sum_{\{j \in \Lambda:|i-j|=1\}}$.

For simplicity we assume that $U(q)=\bar{U}\left(|q|^{2}\right), \bar{U} \in \mathcal{C}^{\infty}(\mathbb{R}, \mathbb{R}), \bar{U}(0)=0$ and $c^{-1} \leq \bar{U}^{\prime} \leq c$ for some finite positive constant $c$; in particular, this implies that $U$ is radially symmetric and strictly convex 1 Also, we assume $\|\nabla V(q)\|^{2} \leq c U(q)$ and $V(-q)=V(q)$.

For simplicity of notation, we choose $\nu=2$. All results stated in this paper are valid for general $\nu \geq 2$, with slight modifications of notation.

In addition to the Hamiltonian dynamics, we consider random forces that conserve the single-site kinetic energies, given by independent diffusions on the spheres $\left\|p_{i}\right\|^{2}=$ const. In order to define such diffusions, consider the vector fields

$$
X_{i}:=p_{i}^{1} \partial_{p_{i}^{2}}-p_{i}^{2} \partial_{p_{i}^{1}}=: J p_{i} \cdot \partial_{p_{i}}, \quad J=\left(\begin{array}{cc}
0 & -1 \\
1 & 0
\end{array}\right)
$$

and the second-order operator

$$
S=\sum_{i \in \Lambda} X_{i}^{2}
$$

The generator of the process we are interested in is then given by

$$
L_{\varepsilon, \Lambda}=: A_{\varepsilon}+\sigma^{2} S,
$$

where $A_{\varepsilon}=\left\{H_{\varepsilon}^{\Lambda}, \cdot\right\}$ is the usual Hamiltonian operator and $\sigma>0$ measures the strength of the noise. Clearly, $L_{\varepsilon, \Lambda}$ is the generator of a contraction semigroup $P_{\varepsilon}^{t}$ in $L^{2}\left(\mathbb{R}^{2 N \nu}, m_{\varepsilon}\right)$ with stationary measure $m_{\varepsilon}$ and $P_{\varepsilon}^{t} H_{\varepsilon}^{\Lambda}=H_{\varepsilon}^{\Lambda}$, for all $t \in \mathbb{R}_{+}$. Next, we must specify the initial conditions.

The Gibbs measures at temperature $\beta^{-1}$ are defined by

$$
m_{\varepsilon}^{\beta}(d q, d q)=Z_{\varepsilon}(\beta) e^{-\beta H_{\varepsilon}^{\Lambda}(q, p)} d p d q
$$

and are the stationary (equilibrium) probability measures for the dynamics (the canonical ensemble). As a reference measure we pick the one corresponding to $\beta=1$ and we denote it by $m_{\varepsilon}$. Notice that for $\varepsilon$ small enough, $m_{\varepsilon}$ and the product measure $m_{0}$ are equivalent. To simplify notation, we also assume that $U$ is such that

$$
Z_{0}(1)=1=\int_{\mathbb{R}^{4}} e^{-\left(p^{2} / 2+U(q)\right)} d p d q .
$$

We assume that the system is started in an initial distribution $d \nu_{0}:=F_{\varepsilon} d m_{\varepsilon}=$ $F_{0} d m_{0}$.

\footnotetext{
${ }^{1}$ The general, nonradial, case can be treated exactly in the same way at the only price of a much messier algebra. On the contrary, the nonconvex case could hold interesting surprises and hopefully will be investigated in the future.
} 
Assumption 1. We assume that $F_{0} \in L^{2}\left(\mathbb{R}^{4 N}, m_{0}\right)$.

For each $T>0$, the Markov process just described defines a probability on $\Omega=\mathcal{C}^{0}\left([0, T], \mathbb{R}^{2 \nu|\Lambda|}\right)$. We will use $\omega_{t}=(q(t), p(t))$ to designate the elements of $\Omega$ at time $t$.

Remark 2.1. In the following we will suppress the subscripts and superscripts $\Lambda$, when this does not create confusion.

Remark 2.2. Here we have free boundary conditions. It should be possible to treat more general stochastic boundary conditions (e.g. having the particle at the boundary perform an Ornstein-Uhlenbeck process at a given temperature) by the same method. We avoid such a generalization to simplify the presentation.

\section{The Results}

The single-particle energies are

$$
\mathcal{E}_{i}^{\varepsilon}(q, p)=\frac{1}{2}\left\|p_{i}\right\|^{2}+U\left(q_{i}\right)+\frac{1}{2} \varepsilon \sum_{|i-j|=1} V\left(q_{i}-q_{j}\right) .
$$

The time evolution of these energies is given by

$$
\frac{d \mathcal{E}_{i}^{\varepsilon}}{d t}=\varepsilon \sum_{k:|i-k|=1} j_{i, k}
$$

where the energy currents are defined by

$$
j_{i, k}=\frac{1}{2} \nabla V\left(q_{i}-q_{k}\right) \cdot\left(p_{i}+p_{k}\right)
$$

Note that $j_{i, k}=-j_{k, i}$ and that they are functions of the $q_{i}, p_{i}, q_{k}, p_{k}$ only.

If $\varepsilon=0$ the dynamics is given by noninteracting oscillators, and consequently the energy of each oscillator is a conserved quantity. So for $\varepsilon=0$ there is a family of equilibrium measure parametrized by the vector $\underline{a}=\left(a_{i}\right)_{i \in \Lambda}$ of the energy of each oscillator. This is given by $\mu_{\underline{a}}^{\Lambda}$, the microcanonical measure associated to the Hamiltonian flow $H_{0}^{\Lambda}$ on the surface

$$
\Sigma_{\underline{a}}:=\left\{q, p: a_{i}=\mathcal{E}_{i}^{0}(q, p)=\frac{1}{2}\left\|p_{i}\right\|^{2}+U\left(q_{i}\right)\right\}=\mathrm{X}_{i \in \Lambda} \Sigma_{a_{i}} .
$$

Clearly, letting $\mu_{a}$ be the microcanonical measure on the 3-dimensional surface $\Sigma_{a}$, we have $\mu_{\underline{a}}^{\Lambda}=\bigotimes_{i \in \Lambda} \mu_{a_{i}}$. By the symmetry between $p$ and $-p$ it follows that $\mu_{a}\left(j_{i, k}\right)=0$ for each $\underline{a}$.

We are interested in the random variables determined by the time rescaled energies

$$
\mathcal{E}_{i}^{\varepsilon}(t)=\mathcal{E}_{i}^{\varepsilon}\left(q\left(\varepsilon^{-2} t\right), p\left(\varepsilon^{-2} t\right)\right) .
$$

In order to define the parameters of the mesoscopic evolution, consider the dynamics of two noninteracting oscillators $(\varepsilon=0)$, each starting with the microcanonical distribution with corresponding energy $a_{1}$ and $a_{2}$. Let us denote by $\mathbb{E}_{a_{1}, a_{2}}(\cdot)$ the corresponding expectation in this equilibrium measure. We will show that the following function on $\mathbb{R}_{+}^{2}$,

$$
\gamma^{2}\left(a_{1}, a_{2}\right)=\int_{0}^{\infty} \mathbb{E}_{a_{1}, a_{2}}\left(j_{1,2}(t) j_{1,2}(0)\right) d t
$$


is well defined. Furthermore, in Lemma 8.2 , we prove that

$$
\gamma^{2}\left(a_{1}, a_{2}\right)=a_{1} a_{2} G\left(a_{1}, a_{2}\right),
$$

where $G$ is a positive symmetric smooth function. Correspondingly we define the mesoscopic current by the antisymmetric function

$$
\alpha\left(a_{1}, a_{2}\right)=e^{\mathcal{U}(\underline{a})}\left(\partial_{a_{1}}-\partial_{a_{2}}\right)\left(e^{-\mathcal{U}(\underline{a})} \gamma^{2}\left(a_{1}, a_{2}\right)\right),
$$

where $\mathcal{U}(\underline{a})=-\sum_{j} \log \mathcal{Z}\left(a_{j}\right)$, and $\mathcal{Z}(a)$ is the energy density distribution under $m_{0}$, which behaves like $a$ for small $a$.

Here is our main result:

Theorem 1. In the limit $\varepsilon \rightarrow 0$, the law of $\left\{\mathcal{E}_{i}^{\varepsilon}\right\}_{i \in \Lambda}$ converges to the weak solution of the stochastic differential equations

$$
d \mathcal{E}_{i}=\sum_{k:|i-k|=1} \alpha\left(\mathcal{E}_{i}, \mathcal{E}_{k}\right) d t+\sum_{k:|i-k|=1} \sqrt{2} \gamma\left(\mathcal{E}_{i}, \mathcal{E}_{k}\right) d B_{\{i, k\}}
$$

with $B_{\{i, k\}}=-B_{\{k, i\}}$ independent standard Brownian motions, where the law of $\mathcal{E}_{i}(0)$ is given by the marginal of $F_{\varepsilon} d m_{\varepsilon}$ on the $\mathcal{E}^{\varepsilon}$.

Notice that the generator of the diffusion (3.8) on $\mathbb{R}_{+}^{\Lambda}$ is given by

$$
\mathcal{L}=\frac{1}{2} \sum_{|k-i|=1}\left(\gamma\left(\mathcal{E}_{i}, \mathcal{E}_{k}\right)^{2}\left(\partial_{\mathcal{E}_{i}}-\partial_{\boldsymbol{E}_{k}}\right)^{2}+\alpha\left(\mathcal{E}_{i}, \mathcal{E}_{k}\right)\left(\partial_{\mathcal{E}_{i}}-\partial_{\boldsymbol{E}_{k}}\right)\right) .
$$

Since $\gamma^{2}$ and $\alpha$ are smooth functions on $\mathbb{R}_{+}^{2}$, the uniqueness of the weak solution of (3.8) follows by applying the results in [3].

Observe that there is a family of product probability measures

$$
\prod_{i \in \Lambda} \mathcal{Z}\left(a_{i}\right) e^{-\beta a_{i}} N(\beta)^{-1} d a_{i}=\prod_{i \in \Lambda} e^{-\left(\beta a_{i}+\mathcal{U}\left(a_{i}\right)\right)} N(\beta)^{-1} d a_{i}, \quad \beta>0
$$

that are stationary and reversible for the diffusion generated by (3.9).

As we will see shortly, the proof of Theorem 1 relies heavily on the fact that the unperturbed microscopic dynamics $P_{\Lambda}^{t}$ generated by $L_{0, \Lambda}$ has strong mixing properties (hypocoercivity). This is itself a nontrivial result, which we believe worth stating separately. Let $\mathcal{H}_{\underline{a}}^{1}$ be the Sobolev space of order one on $\Sigma_{\underline{a}}$ with respect to the microcanonical measure $\mu_{\underline{a}}$ and a properly rescaled Riemannian structure (see Section 6 for more details).

Theorem 2. For each set $\Lambda \subset \mathbb{Z}^{d}$, there exists $C, \tau>0$ such that, for all energies $\underline{a} \in(0, \infty)^{\Lambda}$ and $\sigma \in(0,1)$, the following hold true:

- The semigroup $P_{0, \Lambda}^{t}$ is contractive in $L^{2}\left(\Sigma_{\underline{a}}, \mu_{\underline{a}}\right)$.

- For each smooth function $f \in \mathcal{H}_{a}^{1}$ such that $\mu_{\underline{a}}(f)=0$ holds,

$$
\left\|P_{0, \Lambda}^{t} f\right\|_{\mathcal{H}_{\underline{a}}^{1}} \leq C e^{-\tau \sigma^{2} t}\|f\|_{\mathcal{H}_{\underline{a}}^{1}} .
$$

The proof is given in Section 6 .

Before discussing the proof of the above results, let us indulge in several remarks.

Remark 3.1. By (3.7) we can rewrite the generator as

$$
\mathcal{L}=\frac{1}{2} \sum_{|k-i|=1} e^{\mathcal{U}(\underline{\boldsymbol{\varepsilon}})}\left(\partial_{\boldsymbol{\varepsilon}_{i}}-\partial_{\boldsymbol{\varepsilon}_{k}}\right) e^{-\mathcal{U}(\underline{\boldsymbol{\varepsilon}})} \gamma^{2}\left(\mathcal{E}_{i}, \mathcal{E}_{k}\right)\left(\partial_{\boldsymbol{\varepsilon}_{i}}-\partial_{\boldsymbol{\varepsilon}_{k}}\right)
$$


Remark 3.2. The process (3.8) is close to the one studied by Varadhan in [14, yet it is not covered by such results (due to the degeneracy at zero of the diffusion coefficients and the nonstrict convexity of the potential of the invariant measure). In any case, the extension of Varadhan's work to the present case would allow us to obtain the heat equation in the present setting via a diffusive scaling limit of space and time (hydrodynamic limit).

Remark 3.3. Note that both $\gamma$ and $\alpha$ depend on $\sigma$. One can wonder if equation (3.8) does admit a limit for small noise. Indeed, if $U$ and $V$ are quadratic, then both $\gamma^{2}$ and $\alpha$ are proportional to $\sigma^{-2}$; see (E.4). Hence the energy exchange for small noise is faster than the time scale we are exploring. This is due to the fact that in the quadratic case the solutions are quasiperiodic. On the other hand, for each $U, V$ a positive measure of such quasiperiodic solutions will survive by the KAM theorem at least for small energies, so it may be possible that a small noise limit exists, upon rescaling time, as in the quadratic case. The present results allow only upper bounds (which agree with the quadratic case), but it is unclear if a sufficiently exact scaling still exists.

\section{Proof of MAin theorem}

This section is devoted to proving Theorem 1 by using several results detailed in the later sections (more precisely we assume Proposition 6.1 and Lemmata 7.3. 7.4. 8.1 and 8.2). Our strategy is the first establish tightness and then to show that the accumulation points satisfy (3.8). Since (3.8) has a unique solution, the process has a unique accumulation point, whereby proving the existence of the limit.

4.1. Tightness. Here we start studying the processes $\left\{\mathcal{E}_{i}^{\varepsilon}(t)\right\}$ defined in (3.4).

Lemma 4.1. There exists $\varepsilon_{0}>0$ such that for each $T>0$, the processes $\left\{\mathcal{E}_{i}^{\varepsilon}(t)\right\}_{t \leq T}$, $0 \leq \varepsilon \leq \varepsilon_{0}$, are tight.

Proof. The proof uses a standard backward/forward martingale decomposition argument (cf. [13, 9]). We recall it here.

Let us start the process with the equilibrium distribution $m_{\varepsilon}$. Then the timereversed process, in a given time interval, is a Markov process with generator given by the adjoint $L_{\varepsilon}^{*}=-A_{\varepsilon}+\sigma^{2} S$. Observe that, since $X_{k}^{2} p_{k}=-p_{k}$, we have $S j_{i, k}=$ $-j_{i, k}$. So we can decompose

$$
\begin{aligned}
\varepsilon \int_{0}^{t \varepsilon^{-2}} j_{i, k}(s) d s=-\frac{\varepsilon}{2 \sigma^{2}} \int_{0}^{t \varepsilon^{-2}} L_{\varepsilon} j_{i, k}(s) d s & -\frac{\varepsilon}{2 \sigma^{2}} \int_{0}^{t \varepsilon^{-2}} L_{\varepsilon}^{*} j_{i, k}(s) d s \\
& =\frac{\varepsilon}{2 \sigma^{2}} M_{t \varepsilon^{-2}}^{+}+\frac{\varepsilon}{2 \sigma^{2}} M_{t \varepsilon^{-2}}^{-},
\end{aligned}
$$

where the $M_{t}^{ \pm}$are continuous martingales, adapted respectively to the forward and backward filtration, which can be represented by the stochastic integrals

$$
M_{t}^{ \pm}=\int_{0}^{t}\left(X_{i} j_{j, k}\right)(s) d w_{i}^{ \pm}(s)+\int_{0}^{t}\left(X_{k} j_{j, k}\right)(s) d w_{k}^{ \pm}(s),
$$

where $w_{i}^{+}(t)$ and $w_{i}^{-}(t)$ are standard Wiener processes adapted respectively to the forward and the backward filtration. Consequently the tightness follows from the 
tightness of each of these stochastic integrals. Noticing that $X_{k} j_{j, k}=J p_{k} \cdot \nabla V\left(q_{j}-\right.$ $\left.q_{k}\right) / 2$ is in $L^{p}\left(m_{\varepsilon}\right)$ for any $p<\infty$, by Doob's inequality:

$$
\mathbb{E}_{m_{\varepsilon}}\left(\sup _{0 \leq t \leq T}\left(M_{t}^{ \pm}\right)^{4}\right) \leq\left(\frac{4}{3}\right)^{4} \mathbb{E}_{m_{\varepsilon}}\left(\left(M_{T}^{ \pm}\right)^{4}\right) \leq C T^{2} .
$$

This implies that the Kolmogorov criterion for tightness is satisfied:

$$
\sup _{\varepsilon} \mathbb{E}_{m_{\varepsilon}}\left(\left|\mathcal{E}_{i}^{\varepsilon}(s)-\mathcal{E}_{i}^{\varepsilon}(t)\right|^{4}\right) \leq \frac{C^{\prime}}{\sigma^{4}}(t-s)^{2} .
$$

In nonequilibrium, because of the assumption that the initial distribution $F$ is in $L^{2}\left(m_{\varepsilon}\right)$, the above argument extends immediately by a simple use of the Schwarz inequality.

Observe that with the same argument we can also establish the following bound for any $p>1$ :

$$
\mathbb{E}_{m_{\varepsilon}}\left(\sup _{0 \leq t \leq T}\left[\int_{0}^{t} j_{i, k}(s) d s\right]^{p}\right) \leq \frac{C T^{p / 2}}{\sigma^{p}},
$$

where $C$ is a constant independent of $\varepsilon, T, \sigma$.

Once we have the tightness, all that is left to prove is that the limit is unique. This is the content of the next section.

4.2. Identification of the limit. The goal of this section is to prove that any accumulation point of the laws of $\left\{\mathcal{E}_{i}^{\varepsilon}(t)\right\}_{i \in \Lambda}$ must satisfy equation (3.8). More precisely, since

$$
\mathcal{E}_{i}^{\varepsilon}(t)-\mathcal{E}_{i}^{\varepsilon}(0)=\sum_{k:|k-i|=1} \varepsilon \int_{0}^{\varepsilon^{-2} t} j_{i, k}(s) d s,
$$

we want to show that, for every couple $i, k \in \Lambda$ such that $|i-k|=1$, there exist orthogonal martingales $\mathcal{M}_{i, k}^{\varepsilon}=-\mathcal{M}_{k, i}^{\varepsilon}$ with zero average and quadratic variance given by

$$
2 \int_{0}^{t} \gamma^{2}\left(\mathcal{E}_{i}^{\varepsilon}(s), \mathcal{E}_{k}^{\varepsilon}(s)\right) d s
$$

and such that, for each $t \geq 0$,

$$
\lim _{\varepsilon \rightarrow 0} \mathbb{E}_{F}^{\varepsilon}\left(\left|\varepsilon \int_{0}^{\varepsilon^{-2} t} j_{i, k}(s) d s-\int_{0}^{t} \alpha\left(\mathcal{E}_{i}^{\varepsilon}(s), \mathcal{E}_{k}^{\varepsilon}(s)\right) d s+\mathcal{M}_{i, k}^{\varepsilon}(t)\right|\right)=0 .
$$

To prove (4.4) it turns out to be useful to introduce a cutoff for high energy $\xi_{K}=\chi_{K}\left(\mathcal{E}^{0}\right)$, where $\chi_{K}: \mathbb{R}_{+}^{\Lambda} \rightarrow[0,1]$ are smooth positive functions with support in $[0, K+1]^{\Lambda}$, such that $\chi_{K}(a)=1$ if $a \in[0, K]^{\Lambda}$. Then define $j_{i, k}^{K}=j_{i, k} \xi_{K}$.

By the symmetry between $p$ and $-p$ it follows that $\mu_{\underline{a}}\left(j_{i, k}^{K}\right)=0$ for each $\underline{a} \in \mathbb{R}_{+}^{\Lambda}$ and any $K>0$. Arguing as in estimate (4.1), we have that

$$
\lim _{K \rightarrow \infty} \sup _{\varepsilon>0} \mathbb{E}_{m_{\varepsilon}}\left(\sup _{0 \leq t \leq T}\left[\varepsilon \int_{0}^{t \varepsilon^{-2}} j_{i, k}\left(1-\xi_{K}\right)(s) d s\right]^{2}\right)=0 .
$$

By Proposition 6.1, it follows that the equation

$$
L_{0} u_{i, k}=j_{i, k}
$$

has a unique solution with zero average with respect to all measures $\mu_{\underline{a}}$. Note that $j_{i, k}=-j_{k, i}$; thus also $u_{i, k}=-u_{k, i}$. 
In addition, Lemma 7.3 implies that $u_{i, k} \in \mathcal{C}^{\infty}\left(\left[\mathbb{R}^{4} \backslash\{0\}\right]^{2}, \mathbb{R}\right) \cap \mathcal{C}^{0}\left(\mathbb{R}^{8}, \mathbb{R}\right)$. Observe that, since $L_{0}$ conserves the energies of all particles, denoting $u_{i, j}^{K}=u_{i, j} \xi_{K}$ we have

$$
L_{0} u_{i, k}^{K}=j_{i, k}^{K}
$$

We can thus write $L_{\varepsilon}=L_{0}+\varepsilon L_{*}$ with

$$
L_{*} f=\frac{1}{2} \sum_{|i-j|=1} \nabla V\left(q_{i}-q_{j}\right) \cdot\left(\partial_{p_{i}}-\partial_{p_{j}}\right) f=\sum_{|i-j|=1} \nabla V\left(q_{i}-q_{j}\right) \cdot \partial_{p_{i}} f .
$$

So we have

$$
L_{\varepsilon} u_{i, k}^{K}=j_{i, k}^{K}+\varepsilon L_{\star} u_{i, k}^{K} .
$$

Hence, denoting a path by $\omega_{s}=(q(s), p(s))$, we have

$$
\begin{aligned}
& \varepsilon \int_{0}^{\varepsilon^{-2} t} j_{i, k}^{K}(s) d s=\varepsilon \int_{0}^{\varepsilon^{-2} t}\left[L_{\varepsilon} u_{i, k}^{K}-\varepsilon L_{\star} u_{i, k}^{K}\right]\left(\omega_{s}\right) d s \\
& =\left(\varepsilon u_{i, k}^{K}\left(\omega_{\varepsilon^{-2} t}\right)-\varepsilon u_{i, k}^{K}\left(\omega_{0}\right)-M_{i, k}^{\varepsilon, K}(t)-\varepsilon^{2} \int_{0}^{\varepsilon^{-2} t} L_{\star} u_{i, k}^{K}\left(\omega_{s}\right) d s\right),
\end{aligned}
$$

where we have introduced the centered martingale

$$
M_{i, k}^{\varepsilon, K}(t):=\varepsilon u_{i, k}^{K}\left(\omega_{\varepsilon^{-2} t}\right)-\varepsilon u_{i, k}^{K}\left(\omega_{0}\right)-\varepsilon \int_{0}^{\varepsilon^{-2} t} L_{\varepsilon} u_{i, k}^{K}\left(\omega_{s}\right) d s .
$$

Due to the property $u_{i, k}=-u_{k, i}$ we have $M_{i, k}^{\varepsilon, K}=-M_{k, i}^{\varepsilon, K}$ and the same for all the derived martingales.

The quadratic variations of $M_{i, k}^{\varepsilon, K}$ are given by

$$
\begin{array}{r}
\left\langle\left\langle M_{i, k}^{\varepsilon, K}, M_{i^{\prime}, k^{\prime}}^{\varepsilon, K}\right\rangle(t)=2 \sigma^{2} \sum_{j \in \Lambda} \varepsilon^{2} \int_{0}^{\varepsilon^{-2} t}\left(X_{j} u_{i, k}^{K}\right)\left(X_{j} u_{i^{\prime}, k^{\prime}}^{K}\right)\left(\omega_{s}\right) d s\right. \\
=2 \sigma^{2} \sum_{j \in\{i, k\} \cap\left\{i^{\prime}, k^{\prime}\right\}} \varepsilon^{2} \int_{0}^{\varepsilon^{-2} t} \xi_{K}^{2}\left(X_{j} u_{i, k}\right)\left(X_{j} u_{i^{\prime}, k^{\prime}}\right)\left(\omega_{s}\right) d s .
\end{array}
$$

In order to close the evolution equations, as $\varepsilon \rightarrow 0$, we need to prove that

$$
\lim _{\varepsilon \rightarrow 0} \mathbb{E}_{m_{\varepsilon}}^{\varepsilon}\left|\varepsilon^{2} \int_{0}^{\varepsilon^{-2} t}\left[L_{\star} u_{i, k}^{K}\left(\omega_{s}\right)-\mu_{\underline{\mathcal{E}}^{0}\left(\omega_{s}\right)}\left(L_{*} u_{i, k}^{K}\right)\right] d s\right|^{2}=0
$$

and that

$\lim _{\varepsilon \rightarrow 0} \mathbb{E}_{m_{\varepsilon}}^{\varepsilon}\left|\varepsilon^{2} \int_{0}^{\varepsilon^{-2} t} \xi_{K}^{2}\left[\left(X_{j} u_{i, k}\right)\left(X_{j} u_{i^{\prime}, k^{\prime}}\right)\left(\omega_{s}\right)-\mu_{\underline{\mathcal{E}}^{0}\left(\omega_{s}\right)}\left(\left(X_{j} u_{i, k}\right)\left(X_{j} u_{i^{\prime}, k^{\prime}}\right)\right)\right] d s\right|^{2}=0$.

These are consequences of Lemmas 7.4, 7.3 and the following lemma:

Lemma 4.2. Let $f(\omega)$ be a function in $L^{2}\left(m_{0}\right)$ such that $\mu_{\underline{a}}(f)=0$ for all $\underline{a} \in \mathbb{R}_{+}^{|\Lambda|}$. Then

$$
\lim _{\varepsilon \rightarrow 0} \mathbb{E}_{m_{\varepsilon}}^{\varepsilon}\left|\varepsilon^{2} \int_{0}^{\varepsilon^{-2} t} f\left(\omega_{s}\right) d s\right|^{2}=0
$$


Proof. By using Jensen's inequality and stationarity, it is enough to prove that

$$
\lim _{T \rightarrow \infty} \lim _{\varepsilon \rightarrow 0} \mathbb{E}_{m_{\varepsilon}}^{\varepsilon}\left(\left|\frac{1}{T} \int_{0}^{T} f\left(\omega_{s}\right) d s\right|^{2}\right)=0 .
$$

Since $\lim _{\varepsilon \rightarrow 0} \mathbb{E}_{m_{\varepsilon}}^{\varepsilon}=\mathbb{E}_{m_{0}}^{0}$, i.e., the expectation with respect to the dynamics of the noninteracting oscillators starting with the product of canonical measures $m_{0}$, which are a convex combination of the microcanonical ones, then the result follows by the ergodicity of the dynamics of the single oscillators.

So far we have obtained that

$$
\lim _{\varepsilon \rightarrow 0} \mathbb{E}_{m_{\varepsilon}}^{\varepsilon}\left(\left|\varepsilon^{2} \int_{0}^{\varepsilon^{-2} t}\left(\varepsilon^{-1} j_{i, k}^{K}(s)-\mu_{\underline{\mathcal{E}}^{0}\left(\omega_{s}\right)}\left(L_{\star} u_{i, k}^{K}\right)\right) d s-\hat{M}_{i, k}^{\varepsilon, K}(t)\right|^{2}\right)=0,
$$

where the martingales $\hat{M}_{i, k}^{\varepsilon, K}$ have quadratic variation given by

$$
\left\langle\left\langle\hat{M}_{i, k}^{\varepsilon, K}, \hat{M}_{i^{\prime}, k^{\prime}}^{\varepsilon, K}\right\rangle\right\rangle(t)=\underset{j \in\{i, k\} \cap\left\{\sigma^{\prime}, k^{\prime}\right\}}{2} \xi_{K}\left(\underline{\mathcal{E}}_{0}(s)\right)^{2} \mu_{\underline{\mathcal{E}}_{0}(s)}\left(\left(X_{j} u_{i, k}\right)\left(X_{j} u_{i^{\prime}, k^{\prime}}\right)\right) .
$$

Next, we remove the cutoff on the energies. Observe that

$$
L_{\star} u_{i, k}^{K}=\xi_{K} L_{\star} u_{i, k}+u_{i, k} L_{\star} \xi_{K},
$$

which, writing $\xi_{K}^{(j)}(\underline{a})$ for $\partial_{a_{j}} \xi_{K}(\underline{a})$, implies that

$$
\begin{array}{r}
\mu_{\underline{a}}\left(L_{*} u_{i, k}^{K}\right)=\xi_{K}(\underline{a}) \mu_{\underline{a}}\left(L_{*} u_{i, k}\right)+2 \sum_{|i-j|=1} \xi_{K}^{(j)}(\underline{a}) \mu_{\underline{a}}\left(u_{i, k} \nabla V\left(q_{i}-q_{j}\right) \cdot p_{j}\right) \\
=\xi_{K}(\underline{a}) \mu_{\underline{a}}\left(L_{\star} u_{i, k}\right)+2 \xi_{K}^{(k)}(\underline{a}) \mu_{\underline{a}}\left(u_{i, k} \nabla V\left(q_{i}-q_{k}\right) \cdot p_{k}\right) \\
=\xi_{K}(\underline{a}) \mu_{\underline{a}}\left(L_{*} u_{i, k}\right)+\xi_{K}^{(k)}(\underline{a}) \gamma^{2}\left(a_{i}, a_{k}\right) .
\end{array}
$$

Since $m_{\varepsilon}\left(\mathcal{E}_{j} \geq K\right)$ is exponentially small in $K$, and $\gamma^{2}$ does not grow faster than polynomially, we have

$$
\begin{array}{r}
\sup _{\varepsilon} \mathbb{E}_{m_{\varepsilon}}^{\varepsilon}\left(\left|\varepsilon^{2} \int_{0}^{\varepsilon^{-2} t}\left(\mu_{\underline{\mathcal{E}}^{0}\left(\omega_{s}\right)}\left(L_{\star} u_{i, k}^{K}\right)-\xi_{K}\left(\underline{\mathcal{E}}^{0}\left(\omega_{s}\right)\right) \mu_{\underline{\mathcal{E}}^{0}\left(\omega_{s}\right)}\left(L_{\star} u_{i, k}\right)\right) d s\right|^{2}\right) \\
\leq C \sup _{\varepsilon} \sum_{j} m_{\varepsilon}\left(\gamma^{2}\left(\mathcal{E}_{i}^{0}, \mathcal{E}_{k}^{0}\right) \mathbb{1}_{\left[\mathcal{E}_{j} \in(K, K+1)\right]}\right) K^{2} \underset{K \rightarrow \infty}{\longrightarrow} 0 .
\end{array}
$$

Since the above limit and (4.5) are uniform in $\varepsilon$, and since $X_{j} u_{i, k}$ is in $L^{2}\left(m_{\varepsilon}\right)$, we can take the limit $K \rightarrow \infty$ in (4.15) and obtain

$$
\lim _{\varepsilon \rightarrow 0} \mathbb{E}_{m_{\varepsilon}}^{\varepsilon}\left(\left|\varepsilon \int_{0}^{\varepsilon^{-2} t} j_{i, k}(s) d s-\varepsilon^{2} \int_{0}^{\varepsilon^{-2} t} \mu_{\underline{\mathcal{E}}^{0}\left(\omega_{s}\right)}\left(L_{*} u_{i, k}\right) d s+M_{i, k}^{\varepsilon}(t)\right|^{2}\right)=0
$$

where $M_{i, k}^{\varepsilon}(t)$ is a martingale whose quadratic variation has the expression given by (4.16) with $\xi_{K}$ substituted by 1 .

Lemma 4.3. For each $a_{k}, q_{i}, p_{i}$, setting

$$
\bar{u}_{i}\left(q_{i}, p_{i}\right):=\int u_{k, i}\left(q_{k}, p_{k}, q_{i}, p_{i}\right) \mu_{a_{k}}\left(d q_{k}, d p_{k}\right),
$$

the following holds true:

$$
\bar{u}_{i}\left(q_{i}, p_{i}\right)=\int V\left(q_{i}-q_{k}\right) \mu_{a_{k}}\left(d q_{k}, d p_{k}\right)-\int V\left(q_{i}^{\prime}-q_{k}\right) \mu_{a_{k}}\left(d q_{k}, d p_{k}\right) \mu_{\mathcal{E}_{i}^{0}}\left(d q_{i}^{\prime}, d p_{i}^{\prime}\right) .
$$

In particular, $\bar{u}_{i}\left(q_{i},-p_{i}\right)=\bar{u}_{i}\left(q_{i}, p_{i}\right)$ and $X_{i} \bar{u}_{i}=0$. 
Proof. First of all note that, due to the symmetry between $p$ and $-p$,

$$
\int j_{k, i}\left(q_{k}, p_{k}, q_{i}, p_{i}\right) \mu_{a_{k}}\left(d q_{k}, d p_{k}\right)=\int \partial_{q_{i}} V\left(q_{i}-q_{k}\right) p_{i} \mu_{a_{k}}\left(d q_{k}, d p_{k}\right) .
$$

Thus, setting $\bar{V}\left(q_{i}\right)=\int V\left(q_{i}-q_{k}\right) \mu_{a_{k}}\left(d q_{k}, d p_{k}\right)$,

$$
\int j_{k, i}\left(q_{k}, p_{k}, q_{i}, p_{i}\right) \mu_{a_{k}}\left(d q_{k}, d p_{k}\right)=L_{0,\{i\}} \bar{V}\left(q_{i}, p_{i}\right)
$$

In addition, by the product structure of the generator,

$$
j_{k, i}=L_{0, \Lambda} u_{k, i}=L_{0,\{i\}} u_{k, i}+L_{0,\{k\}} u_{k, i} .
$$

Integrating the above we have

$$
L_{0,\{i\}} \bar{V}\left(q_{i}, p_{i}\right)=\int\left[L_{0,\{i\}} u_{k, i}+L_{0,\{k\}} u_{k, i}\right] \mu_{a_{k}}\left(d q_{k}, d p_{k}\right) .
$$

Since $\mu_{a_{k}}$ is the invariant measure of $L_{0,\{k\}}$, we have

$$
L_{0,\{i\}} \bar{V}\left(q_{i}, p_{i}\right)=L_{0,\{i\}} \bar{u}_{i} .
$$

By Proposition 6.1 applied with $\Lambda=\{i\}$ it follows that the only solutions of the above equation are of the form $\bar{u}_{i}=\bar{V}+f\left(\mathcal{E}_{i}^{0}\right)$ for some function $f$. Next, since $\bar{u}_{i}$ is of zero average by construction, $f\left(\mathcal{E}_{i}^{0}\right)=-\mu_{\mathcal{E}_{i}^{0}}(\bar{V})$.

Thus, if $l \notin\{i, k\}$ we have

$$
\begin{array}{r}
\iiint \partial_{p_{i}} u_{i, k} \cdot \nabla V\left(q_{i}-q_{l}\right) \mu_{a_{k}}\left(d q_{k}, d p_{k}\right) \mu_{a_{i}}\left(d q_{i}, d p_{i}\right) \mu_{a_{l}}\left(d q_{l}, d p_{l}\right) \\
=\iint \partial_{p_{i}} \bar{u}_{i} \cdot \nabla V\left(q_{i}-q_{l}\right) \mu_{a_{i}}\left(d q_{i}, d p_{i}\right) \mu_{a_{l}}\left(d q_{l}, d p_{l}\right)=0
\end{array}
$$

due to the antisymmetry of $\partial_{p_{i}} \bar{u}_{i}$ with respect to $p_{i}$ established in Lemma 4.3 . From this it follows that

$$
\mu_{\underline{a}}\left(L_{\star} u_{i, k}\right)=\mu_{\underline{a}}\left[\nabla V\left(q_{i}-q_{k}\right)\left(\partial_{p_{i}}-\partial_{p_{k}}\right) u_{i, k}\right]=: \alpha\left(a_{i}, a_{k}\right) .
$$

In fact, again by the product structure, the above is a function of $a_{i}, a_{k}$ only.

It is also convenient to define

$$
\gamma^{2}\left(a_{i}, a_{k}\right)=\sigma^{2} \mu_{\underline{a}}\left(\left(X_{i} u_{i, k}\right)^{2}+\left(X_{k} u_{i, k}\right)^{2}\right)=-\mu_{\underline{a}}\left(u_{i, k} j_{i, k}\right) .
$$

Accordingly, by (4.16) and Lemma 4.3.

$$
\left\langle\left\langle\hat{M}_{i, k}^{\varepsilon}, \hat{M}_{i^{\prime}, k^{\prime}}^{\varepsilon}\right\rangle\right\rangle(t)=2 \sigma^{2} \sum_{j \in\{i, k\}} \mu_{\underline{\mathcal{E}}_{0}(s)}\left(\left(X_{j} u_{i, k}\right)^{2}\right)\left(\delta_{i=i^{\prime}, k=k^{\prime}}-\delta_{i=k^{\prime}, k=i^{\prime}}\right) .
$$

\section{Lemma 4.4.}

$$
\lim _{\varepsilon \rightarrow 0} \mathbb{E}_{m_{\varepsilon}}\left|\int_{0}^{t}\left(\alpha\left(\mathcal{E}_{i}^{0}\left(\omega_{\varepsilon^{-2} s}\right), \mathcal{E}_{k}^{0}\left(\omega_{\varepsilon^{-2} s}\right)\right)-\alpha\left(\mathcal{E}_{i}^{\varepsilon}(s), \mathcal{E}_{k}^{\varepsilon}(s)\right)\right) d s\right|^{2}=0
$$

and similarly for $\gamma^{2}$.

Proof. By stationarity and the Schwarz inequality we have

$$
\begin{aligned}
\mathbb{E}_{m_{\varepsilon}} & \left|\int_{0}^{t}\left[\alpha\left(\mathcal{E}_{i}^{0}\left(\omega_{\varepsilon^{-2} s}\right), \mathcal{E}_{k}^{0}\left(\omega_{\varepsilon^{-2} s}\right)\right)-\alpha\left(\mathcal{E}_{i}^{\varepsilon}(s), \mathcal{E}_{k}^{\varepsilon}(s)\right)\right] d s\right|^{2} \\
& \leq t m_{\varepsilon}\left(\left|\alpha\left(\mathcal{E}_{i}^{0}, \mathcal{E}_{k}^{0}\right)-\alpha\left(\mathcal{E}_{i}^{\varepsilon}, \mathcal{E}_{k}^{\varepsilon}\right)\right|^{2}\right) .
\end{aligned}
$$

Since we prove in Lemmata 8.1, 8.2 that $\alpha\left(a_{1}, a_{2}\right)$ is uniformly Lipschitz in $\mathbb{R}_{+}^{2}$ the result follows by elementary arguments. 
Applying Lemma 4.4 to (4.17) and (4.20) we obtain that

$$
\lim _{\varepsilon \rightarrow 0} \mathbb{E}_{m_{\varepsilon}}^{\varepsilon}\left(\left|\varepsilon \int_{0}^{\varepsilon^{-2} t} j_{i, k}(s) d s-\int_{0}^{t} \alpha\left(\mathcal{E}_{i}^{\varepsilon}(s), \mathcal{E}_{k}^{\varepsilon}(s)\right) d s+\mathcal{M}_{i, k}^{\varepsilon}(t)\right|^{2}\right)=0,
$$

which, remembering (4.19), yields the wanted result in equilibrium. Our general claim (4.4) follows by the Schwarz inequality.

\section{HyPOELLIPTICITY AND REGULARITY ON ENERGY SHELLS}

We start here by studying the single-site dynamics. Here $p=\left(p^{1}, p^{2}\right), q=\left(q^{1}, q^{2}\right)$ will be the coordinates. We will use $L$ to designate the generator (2.1) for $\varepsilon=0$ and $\Lambda$ consisting of only one site. Since $L$ preserves the energy, we can look at its action on each energy surface:

$$
\Sigma_{a}=\left\{(q, p) \in \mathbb{R}^{4}: \frac{|p|^{2}}{2}+U(q)=a\right\}, \quad a>0 .
$$

Lemma 5.1. For each $a>0$ the operator $L$ is hypoelliptic on $\Sigma_{a}$.

Proof. We must study the Lie algebra generated by the vector fields

$$
\begin{aligned}
C_{0} & =X=J p \cdot \partial_{p}, \\
B & =A_{0}=p \cdot \partial_{q}-\nabla U(q) \cdot \partial_{p} .
\end{aligned}
$$

We obtain

$$
\begin{gathered}
C_{1}=:\left[C_{0}, B\right]=J p \cdot \partial_{q}+J \nabla U(q) \cdot \partial_{p}, \\
{\left[C_{1}, B\right]=2 J \nabla U(q) \cdot \partial_{q}-\left\{D^{2} U(q) J+J D^{2} U(q)\right\} p \cdot \partial_{p} .}
\end{gathered}
$$

By our assumption on $U$,

$$
D^{2} U(q)=4 \bar{U}^{\prime \prime}(q) q \otimes q+2 \bar{U}^{\prime}(q) \mathbb{1} .
$$

Since

$$
J(q \otimes q)+(q \otimes q) J=|q|^{2} J
$$

we have

$$
D^{2} U(q) J+J D^{2} U(q)=4\left\{\bar{U}^{\prime \prime}(q)|q|^{2}+\bar{U}^{\prime}\right\} J=: \zeta(q) J .
$$

Finally we define $C_{2}$ by the relation

$$
\left[C_{1}, B\right]=4 \bar{U}^{\prime}(q) J q \cdot \partial_{q}-4\left\{\bar{U}^{\prime \prime}(q)|q|^{2}+\bar{U}^{\prime}\right\} J p \cdot \partial_{p}=: 2 C_{2}-\zeta(q) C_{0} .
$$

Observe that, setting $\mathcal{N}=\left(|p|^{2}+|\nabla U(q)|^{2}\right)^{1 / 2}$, the vectors

$$
Z_{1}=\frac{C_{1}}{\mathcal{N}}, \quad Z_{0}=\frac{B}{\mathcal{N}}, \quad Z_{2}=\frac{C_{2}-C_{0}}{\mathcal{N}}
$$

form an orthonormal basis of the tangent space of $\Sigma_{a}$; hence the Lie algebra generated by $\left\{C_{0}, B\right\}$ spans the space of any energy shell $\Sigma_{a}$. This concludes the proof of the hypoellipticity of $L$.

By the above results trivially follows the claimed hypoellipticity.

Lemma 5.2. For each $\Lambda \subset \mathbb{Z}^{d}, a_{i}>0, i \in \Lambda$, the operator $L_{0, \Lambda}$ is hypoelliptic on $\Sigma_{\underline{a}}$. 
By the Hörmander theorem [7, hypoellipticity implies that if there exists a solution $u$ for the equation

$$
L_{0, \Lambda} u=g,
$$

where $g$ is a $\mathcal{C}^{\infty}$ function when restricted to any energy shell, then also $u$ is $\mathcal{C}^{\infty}$ when restricted to any energy shell.

\section{HyPOCOERCIVITY}

We will prove the existence of the solution of equation (4.6) by proving a spectral gap for the generator $L_{0}$ on each energy shell $\Sigma_{\underline{a}}$ in a proper Hilbert space. More precisely we consider the Hilbert spaces determined by the scalar products

$$
\begin{aligned}
\langle h, g\rangle_{\mathscr{H}_{\underline{a}}^{1}} & :=|\Lambda|\langle h, g\rangle_{\underline{a}}+\sum_{l \in \Lambda} \mathcal{D}_{\underline{a}, l}(h, g), \\
\mathcal{D}_{\underline{a}, l}(h, g) & :=\sum_{k=0}^{2}\left\langle C_{k, l} h, C_{k, l} g\right\rangle_{\underline{a}}+\left\langle B_{l} h, B_{l} g\right\rangle_{\underline{a}},
\end{aligned}
$$

where $\langle h, g\rangle_{\underline{a}}=\int_{\Sigma_{\underline{a}}} \bar{h} g d \mu_{\underline{a}}$ and the $C_{k, l}, B_{l}$ are the vector fields defined in Section 5 relative to the particle $\left(q_{l}, p_{l}\right)$.

By a slight abuse of notation we will use $P_{0}^{t}$ to denote the strongly continuous semigroup generated by $L_{0}$ both in $L^{2}$ and $\mathscr{H}_{\underline{a}}^{1}$ for each $\underline{a}$.

Note that the above norm is equivalent to the standard Sobolev space $\mathcal{H}_{a}$ on the Riemannian surface $\Sigma_{a}$, where the Riemannian structure has been rescaled to have the diameter of each $\bar{\Sigma}_{a_{i}}$ equal one independently of the $a_{i}$. More precisely, there exists $c>0$ such that

$$
c\|f\|_{\mathcal{H}_{\underline{a}}} \leq\|f\|_{\mathscr{H}_{\underline{a}}} \leq c^{-1}|\Lambda|\|f\|_{\mathcal{H}_{\underline{a}}} .
$$

Accordingly, Theorem 2 is a direct consequence of the following.

Proposition 6.1. The semigroup $P_{0}^{t}$ is contractive in $L^{2}$. In addition, for each $\Lambda \subset \mathbb{R}^{d},|\Lambda|<\infty$, there exists $C, \tau>0$ such that for all $\underline{a} \in(0, \infty)^{\Lambda}$ and smooth functions $f$, such that $\mu_{\underline{a}}(f)=0$, it follows that

$$
\left\|P_{0}^{t} f\right\|_{\mathscr{H}_{\underline{a}}^{1}} \leq C e^{-\tau t}\|f\|_{\mathscr{H}_{\underline{a}}^{1}} .
$$

Proof. The contractivity in $L^{2}$ follows by

$$
\frac{d}{d t}\left\|P_{0}^{t} f\right\|_{\underline{a}}^{2} \leq-2 \sum_{L}\left\langle C_{0, l} P_{0}^{t} f, C_{0, l} P_{0}^{t} f\right\rangle_{\underline{a}} \leq 0 .
$$

To prove the second part of the theorem we use the results of Appendix B Note that for all smooth $h, g, \varphi$ we can write

$$
m_{0}\left(\varphi(\underline{\mathcal{E}}) \mu_{\underline{\mathcal{E}}}\left(h C_{0} g\right)\right)=m_{0}\left(\varphi(\underline{\mathcal{E}}) h C_{0} g\right)=-m_{0}\left(\varphi(\underline{\mathcal{E}}) g C_{0} h\right) ;
$$

hence it must be $\mu_{\underline{a}}\left(h C_{0} g\right)=-\mu_{\underline{a}}\left(C_{0} h \cdot g\right)$, and the same for $B$. We can thus use Lemma B.2 applied to, for each $l \in \Lambda$, a scalar product $\langle\cdot \cdot \cdot \cdot\rangle_{\underline{a}, l}$ defined in (B.2) using the operators $C_{k, l}, B_{l}$. By Lemma B.1 such a scalar product is equivalent to the product

$$
\langle h, g\rangle_{\mathscr{H}_{\underline{a}, l}^{1}}:=\langle h, g\rangle_{\underline{a}}+\mathcal{D}_{\underline{a}, l}(h, g) .
$$

Then Lemma B.2 states that

$$
\left\langle h, L_{\{l\}} h\right\rangle_{\underline{a}, l} \leq-\tau \sigma^{2}\left(\mathcal{D}_{\underline{a}, l}(h, h)+\mathcal{D}_{\underline{a}, l}\left(C_{0} h, C_{0} h\right)\right) \leq-\tau \sigma^{2} \mathcal{D}_{\underline{a}, l}(h, h),
$$


where $L_{\{l\}}$ is the generator of the dynamics of the isolated atom $l$, i.e. $L_{0}=$ $\sum_{l \in \Lambda} L_{\{l\}}$.

The last piece of information we need is given by the following Poincaré inequality.

Lemma 6.2. There exist a constant $K_{P}>0$ such that for each $\underline{a}$, if $f \in \mathscr{H}_{\underline{a}}^{1}$, such that $\mu_{\underline{a}}(f)=0$, then

$$
\|f\|_{\underline{a}}^{2} \leq K_{P} \sum_{l \in \Lambda} \mathcal{D}_{\underline{a}, l}(f, f) .
$$

Proof. By the change of variable introduced in Appendix Cone can use the Poincaré inequality for the sphere, for one particle. After that,

$$
\begin{aligned}
\|f\|_{\underline{a}}^{2} & \leq\left\|f-\mu_{a_{l}}(f)\right\|_{\underline{a}}^{2}+\left\|\mu_{a_{l}}(f)\right\|_{\underline{a}}^{2} \\
& \leq K_{P} D_{\underline{a}, l}(f, f)+\left\|\mu_{a_{l}}(f)-\mu_{a_{l}, a_{j}}(f)\right\|_{\underline{a}}^{2}+\left\|\mu_{a_{l}, a_{j}}(f)\right\|_{\underline{a}}^{2} ;
\end{aligned}
$$

iterating the argument yields the result.

It follows from (6.3) and Lemma 6.2 that

$$
\begin{aligned}
\left\langle\left\langle h, L_{0} h\right\rangle\right. & =\sum_{l, l^{\prime}}\left\langle\left\langle h, L_{\left\{l^{\prime}\right\}} h\right\rangle_{\underline{a}, l} \leq \sum_{l}\left\langle\left\langle h, L_{\{l\}} h\right\rangle_{\underline{a}, l}-\sum_{l \neq l^{\prime}}\left\langle\left\langle C_{0, l^{\prime}} h, C_{0, l^{\prime}} h\right\rangle_{\underline{a}, l}\right.\right.\right. \\
& \leq \sum_{l}\left\langle\left\langle h, L_{\{l\}} h\right\rangle_{\underline{a}, l} \leq-\tau \sigma^{2} \sum_{l} \mathcal{D}_{\underline{a}, l}(h, h)\right. \\
& \leq-\frac{\tau \sigma^{2}}{1+K_{P}|\Lambda|}\|h\|_{\mathscr{H}_{\underline{a}}}^{2} .
\end{aligned}
$$

Accordingly, for each $\underline{a}$ and $h \in \mathscr{H}_{\underline{a}}^{1}$, such that $\mu_{\underline{a}}(h)=0$, we have

$$
\begin{aligned}
\frac{d}{d t}\left\langle\left\langle P_{0}^{t} h, P_{0}^{t} h\right\rangle_{\underline{a}}\right. & =2\left\langle\left\langle P_{0}^{t} h, L_{0} P_{0}^{t} h\right\rangle_{\underline{a}}\right. \\
& \leq-\frac{2 \tau \sigma^{2}}{1+K_{P}|\Lambda|}\left\|P_{0}^{t} h\right\|_{\mathscr{H}_{\underline{a}}^{1}}^{2} \\
& \leq-2 \tau_{1} \sigma^{2}\left\langle\left\langle P_{0}^{t} h, P_{0}^{t} h\right\rangle_{\underline{a}},\right.
\end{aligned}
$$

where, in the last line, we have used first (6.3) and then Lemma B.1. This means that

$$
\left\langle\left\langle P_{0}^{t} h, P_{0}^{t} h\right\rangle_{\underline{a}} \leq\langle\langle h, h\rangle\rangle_{\underline{a}} e^{-2 \tau_{1} \sigma^{2} t}\right.
$$

and, by the equivalence of the norms, there exists $C, \tau^{\prime}>0$ such that

$$
\left\|P_{0}^{t} h\right\|_{\mathscr{H}_{\underline{a}}} \leq C e^{-\tau^{\prime} \sigma^{2} t}\|h\|_{\mathscr{H}_{\underline{a}}^{1}} .
$$

\section{Global Regularity}

Next, we need regularity of the solution of (5.6) also in directions not tangent to the energy surfaces, provided that $g$ is smooth. Again we work first with only one particle. 
7.1. The transversal direction. A natural direction would be given by the normal vector to the energy surfaces

$$
Z_{3}=\frac{p \cdot \partial_{p}+\nabla U(q) \cdot \partial_{q}}{\mathcal{N}}
$$

but, due to the anharmonicity of the potential $U$, it turns out to be more useful to work with the vector field

$$
Y:=\frac{1}{2 \sqrt{\mathcal{E}^{0}(q, p)}}\left\{\frac{1}{2} p \cdot \partial_{p}+\frac{U(q)}{\nabla U(q) \cdot q} q \cdot \partial_{q}\right\} .
$$

Note that $Y$ is a smooth vector field away from zero. Observe that in the harmonic case $\left(\bar{U}(r)=\frac{1}{2} r\right), Y$ is parallel to the normal vector field $Z_{3}$.

The reason why we consider $Y$ is the following. A direct computation shows that $Y \mathcal{E}^{0}=\frac{1}{2} \sqrt{\mathcal{E}^{0}(q, p)}$; hence the vector field is transverse to the energy surface. In addition,

$$
\begin{aligned}
& {\left[Y, C_{0}\right]=0,} \\
& {[Y, L] \mathcal{E}^{0}=-\frac{1}{2} L \sqrt{\mathcal{E}^{0}(q, p)}=0 .}
\end{aligned}
$$

That is, the commutators are vector fields tangent to the constant energy surface. Note that since $U^{\prime \prime}(0)>0$, we can write $\frac{U(q)}{q \cdot \nabla U(q)}=\frac{1}{2}+\kappa\left(|q|^{2}\right)$, for a smooth function $\kappa$ such that $\kappa(0)=0$. An explicit computation yields

$$
[L, Y]=\frac{4\left(\frac{1}{2}+\kappa\right) \bar{U}^{\prime \prime} q^{2}+2 \kappa \bar{U}^{\prime}}{2 \sqrt{\mathcal{E}^{0}(q, p)}} q \cdot \partial_{p}+\frac{1}{2 \sqrt{\mathcal{E}^{0}(q, p)}} p \cdot\left[2 \kappa^{\prime} q \otimes q+\kappa \mathbb{1}\right] \partial_{q} .
$$

Remark 7.1. Note that the vector field $\left(\mathcal{E}^{0}(q, p)\right)^{-\frac{1}{2}}[L, Y]$ is smooth on all $\mathbb{R}^{2 \nu}$, in particular even at zero. This fact will play a crucial role in the following.

7.2. Transversal regularity. The basic idea to prove regularity is to notice that if $L u=g$, then one expects that $L Y u=[Y, L] u+Y g$. Unfortunately, we have only $L^{2}$ bounds for the right-hand side of the above equations; in particular, we do not know if it belongs to $\mathscr{H}^{1}$. Hence, a priori, we do not know if such an equation has a solution in $\mathscr{H}^{1}$. To overcome such a difficulty several direct strategies are possible. For example one could try to prove a spectral gap in Sobolev spaces of higher regularity or to prove that the semigroup maps $L^{2}$ functions into $\mathscr{H}^{1}$ functions. Unfortunately, such results (even if probably true) are not so easy to prove; in particular, the related algebra becomes quickly very messy. Due to this state of affairs we take a bit more indirect route that, without proving explicit bounds, suffices to prove the smoothness. To this end it is convenient to work in coordinates in which all the energy surfaces can be naturally identified.

It is then natural to transform equation (4.6) into the following coordinates. Let $S^{3}:=\left\{x \in \mathbb{R}^{4}:\|x\|=1\right\}$ and $M=\mathbb{R} \times S^{3} \subset \mathbb{R} \times \mathbb{R}^{2} \times \mathbb{R}^{2}, M_{+}=(0, \infty) \times S^{3} \subset M$. Let $\Psi: \mathbb{R}^{4|\Lambda|} \backslash\{0\} \rightarrow M_{+}^{|\Lambda|} \subset \mathbb{R}^{5|\Lambda|}$ be defined by

$$
\Psi(q, p)_{i}=\left(\sqrt{\frac{p_{i}^{2}}{2}+U\left(q_{i}\right)}, \frac{q_{i} \sqrt{U\left(q_{i}\right)}}{\left|q_{i}\right| \sqrt{\frac{p_{i}^{2}}{2}+U\left(q_{i}\right)}}, \frac{p_{i}}{\sqrt{p_{i}^{2}+2 U\left(q_{i}\right)}}\right)=:\left(r_{i}, \xi_{i}, \eta_{i}\right) .
$$

The needed properties of this change of variables are detailed in Appendix $\mathrm{C}$ 
The first key observation is that the problem is now regularized at zero energy. Indeed,

$$
\begin{aligned}
\tilde{j}_{i, k}(\underline{r}, \underline{\xi}, \underline{\eta}) & =j_{i, k} \circ \Psi^{-1}(\underline{r}, \underline{\xi}, \underline{\eta}) \\
& =\frac{1}{\sqrt{2}} \nabla V\left(r_{i} \theta\left(r_{i}^{2} \xi_{i}^{2}\right) \xi_{i}-r_{k} \theta\left(r_{k}^{2} \xi_{k}^{2}\right) \xi_{k}\right) \cdot\left(r_{i} \eta_{i}+r_{k} \eta_{k}\right) .
\end{aligned}
$$

Remark 7.2. Note that $\tilde{j}_{i, k}$ extends naturally to a smooth function on $M^{\Lambda}$. Indeed if $r_{i}<0$, then we can set $\tilde{j}_{i, k}(\underline{r}, \underline{\xi}, \underline{\eta})=\tilde{j}_{i, k}\left(\underline{r}^{\prime}, \underline{\xi^{\prime}}, \underline{\eta}^{\prime}\right)$, where $r_{j}=r_{j}^{\prime}, \xi_{j}=\xi_{j}^{\prime}, \eta_{j}=\eta_{j}^{\prime}$ for all $j \neq i$ and $r_{i}=-r_{i}^{\prime}, \xi_{i}=-\xi_{i}^{\prime}, \eta_{i}=-\eta_{i}^{\prime}$, and the same for $k$.

Note that for each function $\tilde{f} \in \mathcal{C}^{\infty}\left(M^{|\Lambda|}, \mathbb{R}\right), C_{0}(\tilde{f} \circ \Psi)=\left(\tilde{C}_{0} \tilde{f}\right) \circ \Psi$ holds. It follows that the equation $L_{0} f=g$ on $\left(\mathbb{R}^{4} \backslash\{0\}\right)^{|\Lambda|}$ is transformed in the equation $\tilde{L}_{0} \tilde{f}=\tilde{g}$ on $\Psi\left(\left(\mathbb{R}^{4} \backslash\{0\}\right)^{|\Lambda|}\right) \subset M$, where $\tilde{L}_{0}=\sum_{l} \sigma^{2} \tilde{C}_{0, l}^{2}+\tilde{B}_{l}$ and $\tilde{f}=f \circ \Psi^{-1}$, $\tilde{g}=g \circ \Psi^{-1}$.

It is then natural to study the equation on $M$ :

$$
\tilde{L}_{0} \tilde{u}_{i, k}=\tilde{j}_{i, k} .
$$

By the previous discussion the solution of (4.6) in $\left(\mathbb{R}^{4} \backslash\{0\}\right)^{\Lambda}$ is given by $u_{i, k}=$ $\left.\tilde{u}_{i, k}\right|_{M_{+}^{\Lambda}} \circ \Psi$.

The problem of the transversal smoothness is then reduced to studying the smoothness of $\tilde{u}_{i, k}$ in $r$ (see Lemma C.1).

Lemma 7.3. For each $i, k$, the functions $\tilde{u}_{i, k} \in \mathcal{C}^{\infty}\left(M^{\Lambda}, \mathbb{R}\right)$.

Proof. We can consider $\tilde{C}_{i}, \tilde{B}_{i}^{k}$ as vector fields on $S^{3|\Lambda|} 2$ Accordingly, we can define for each $\underline{r} \in \mathbb{R}^{\Lambda}$,

$$
\tilde{L}_{0}(\underline{r})=\sum_{i \in \Lambda}\left\{\sigma^{2} \tilde{C}_{0, i}^{2}+\frac{\sqrt{2}}{\theta\left(r_{i}^{2} \xi_{i}^{2}\right)}\left[\tilde{B}_{i}^{0}+\Gamma\left(r_{i}^{2} \xi_{i}^{2}\right) \tilde{B}_{i}^{1}\right]\right\} .
$$

Then, setting $\tilde{j}_{i, k, \underline{r}}(\underline{\xi}, \underline{\eta}):=\tilde{j}_{i, k}(\underline{r}, \underline{\xi}, \underline{\eta})$ and considering the equation on $S^{3|\Lambda|}$,

$$
\tilde{L}_{0}(\underline{r}) \tilde{u}_{i, k, \underline{r}}=\tilde{j}_{i, k, \underline{r}}
$$

it follows that $\tilde{u}_{i, k}(\underline{r}, \underline{\xi}, \underline{\eta})=\tilde{u}_{i, k, \underline{r}}(\underline{\xi}, \underline{\eta})$. By the previous section we know that, for each $\underline{r}, \tilde{u}_{i, k, \underline{r}} \in \mathcal{C}^{\infty}\left(S^{3|\Lambda|}, \mathbb{R}\right)$. Thus the differentiability boils down to showing that the solutions of (7.7) are differentiable with respect to the parameter $\underline{r}$.

Let us fix $\underline{r}$ and consider the equation 3

$$
\tilde{L}_{0}(\underline{r}) \tilde{v}_{i, k, l, \underline{r}}=\tilde{g}_{i, k, l, \underline{r}},
$$

where

$$
\tilde{g}_{i, k, \underline{r}}(\underline{\xi}, \underline{\eta})=\left(\left[\tilde{Y}_{l}, \tilde{L}_{0}\right] \tilde{u}_{i, k}\right)(\underline{r}, \underline{\xi}, \underline{\eta})+\left(\tilde{Y}_{l} \tilde{j}_{i, k}\right)(\underline{r}, \underline{\xi}, \underline{\eta}) .
$$

Clearly $\tilde{g}_{i, k, l, \underline{r}} \in \mathcal{C}^{\infty}\left(S^{3|\Lambda|}, \mathbb{R}\right)$ for each choice of $i, k, l, \underline{r}$; thus $\tilde{v}_{i, k, l, \underline{r}} \in \mathcal{C}^{\infty}\left(S^{3|\Lambda|}, \mathbb{R}\right)$. We claim that $\partial_{r_{l}} \tilde{u}_{i, k}=\tilde{v}_{i, k, l, \underline{r}}$. Let us prove it.

For each, small, $h \in \mathbb{R}^{\Lambda}$ we can write

$$
\begin{aligned}
\tilde{L}_{0}(\underline{r}+h)\left[\tilde{u}_{i, k, \underline{r}+h}-\tilde{u}_{i, k, \underline{r}}-\sum_{l} \tilde{v}_{i, k, l, \underline{r}} h_{l}\right]= & \tilde{j}_{i, k, \underline{r}+h}-\tilde{j}_{i, k, \underline{r}}-\sum_{l} \tilde{g}_{i, k, l, \underline{r}} h_{l} \\
& -\left[\tilde{L}_{0}(\underline{r}+h)-\tilde{L}_{0}(\underline{r})\right] \tilde{u}_{i, k, \underline{r}} .
\end{aligned}
$$

\footnotetext{
${ }^{2}$ See (C.2) for the definition of $\tilde{B}_{i}^{k}$.

${ }^{3}$ This is nothing else than the formal derivative of (7.7) with respect to $r_{l}$.
} 
An explicit computation shows that the $\mathscr{H}^{1}$ norm of the right-hand side is bounded by a constant (depending in an unknown manner on $\underline{r}$ ) times $o(\|h\|)$. Then by Proposition 6.1 the differentiability of $u_{i, k, \underline{r}}$ follows.

Note that in the above argument the only relevant property of $\tilde{j}_{i, k, \underline{r}}$ is the smoothness on $S^{3|\Lambda|}$ of itself and of its derivatives with respect to $\tilde{Y}_{l}$. Since a direct computation shows that the same properties are enjoyed by $\tilde{g}_{i, k, l, \underline{r}} \underline{4}$ the lemma follows by iterating the above argument.

Lemma 7.4. It holds true that $L_{\star} u_{i, k} \in L_{\text {loc }}^{2}\left(\mathbb{R}^{8}, m_{0}\right)$.

Proof. Recall the definition of $L_{*}$ given by (4.8). Then all we need to prove is that $\frac{\partial \Psi}{\partial p_{i}}$ is in $L_{\mathrm{loc}}^{2}\left(\mathbb{R}^{8}, m_{0}\right)$. It follows from straightforward calculations that the singularities at 0 of $\frac{\partial \Psi}{\partial p_{i}}$ are $m_{0}$ integrable.

\section{Structure And Regularity of $\gamma^{2}$ AND $\alpha$}

Let $u=u_{1,2}$ be the solution of the Poisson equation (4.6). Then, by (4.19) and (D.6), we write

$$
\begin{aligned}
\gamma^{2}\left(a_{1}, a_{2}\right) & =\mu_{a_{1}, a_{2}}\left(j_{1,2} u_{1,2}\right) \\
& =\frac{16 \omega_{4}^{2} a_{1} a_{2}}{\mathcal{Z}\left(a_{1}\right) \mathcal{Z}\left(a_{2}\right)} \int_{S^{3} \times S^{3}} d \sigma\left(\xi_{1}, \eta_{1}\right) d \sigma\left(\xi_{2}, \eta_{2}\right) \Omega(\sqrt{a}, \xi, \eta),
\end{aligned}
$$

where $\sigma$ is the uniform probability measure on the sphere $S^{3}$ and

$$
\begin{aligned}
\Omega(r, \xi, \eta) & :=\frac{\left(j_{1,2} u_{1,2}\right)\left(\Psi^{-1}\left(r_{1}, \xi_{1}, \eta_{1}\right), \Psi^{-1}\left(r_{2}, \xi_{2}, \eta_{2}\right)\right)}{\bar{U}^{\prime}\left(\rho\left(r_{1}^{2} \xi_{1}^{2}\right)\right) \bar{U}^{\prime}\left(\rho\left(r_{2}^{2} \xi_{2}^{2}\right)\right)} \\
& =\frac{\left(\tilde{j}_{1,2} \tilde{u}_{1,2}\right)\left(r_{1}, \xi_{1}, \eta_{1}, r_{2}, \xi_{2}, \eta_{2}\right)}{\bar{U}^{\prime}\left(\rho\left(r_{1}^{2} \xi_{1}^{2}\right)\right) \bar{U}^{\prime}\left(\rho\left(r_{2}^{2} \xi_{2}^{2}\right)\right)} .
\end{aligned}
$$

We have already seen that $\tilde{u}$ satisfies (7.6) and is a smooth function on $M^{2}$. Hence $\Omega \in \mathcal{C}^{\infty}\left(M^{2}, \mathbb{R}\right)$. By Remark 7.2 and the structure of $\tilde{L}_{0}$ (see Lemma C.1), we have

$$
\left(\tilde{j}_{1,2} \tilde{u}_{1,2}\right)\left(-r_{1},-\xi_{1},-\eta_{1}, r_{2}, \xi_{2}, \eta_{2}\right)=\left(\tilde{j}_{1,2} \tilde{u}_{1,2}\right)\left(r_{1}, \xi_{1}, \eta_{1}, r_{2}, \xi_{2}, \eta_{2}\right)
$$

and the same for the second coordinate. By the symmetry of the measure $\sigma$ it follows then that the integral on the right-hand side of (8.1) is an even smooth function of $\sqrt{a_{1}}, \sqrt{a_{2}}$, hence a smooth function of $a_{1}, a_{2}$. This shows that $\gamma^{2} \in \mathcal{C}^{\infty}\left([0, \infty)^{2}, \mathbb{R}\right)$.

We are now in a position to prove the relation between $\alpha$ and $\gamma$ :

Lemma 8.1. For any nearest neighbor couple $\{i, k\}$ :

$$
e^{\mathcal{U}(\underline{a})}\left(\partial_{a_{i}}-\partial_{a_{k}}\right)\left(e^{-\mathcal{U}(\underline{a})} \gamma^{2}\left(a_{i}, a_{k}\right)\right)=\alpha\left(a_{i}, a_{k}\right)
$$

with $\mathcal{U}(\underline{a})=-\sum_{j} \log \mathcal{Z}\left(a_{j}\right)$.

\footnotetext{
${ }^{4}$ The smoothness on $S^{3|\Lambda|}$ follows from the smoothness of $\tilde{u}_{i, k}$. The differentiability with respect to $\tilde{Y}_{l}$ follows by the smoothness of $\tilde{j}_{i, k, \underline{r}}$ and the fact that $\tilde{Y}_{l^{\prime}}\left[\tilde{Y}_{l}, \tilde{L}_{0}\right] \tilde{u}_{i, k}=\left[\tilde{Y}_{l^{\prime}},\left[\tilde{Y}_{l}, \tilde{L}_{0}\right]\right] \tilde{u}_{i, k}+$ $\left[\tilde{Y}_{l}, \tilde{L}_{0}\right] \tilde{v}_{i, k, l^{\prime}, \underline{r}}$, since it is easy to check that, given any vector $Z$ tangent to $M^{|\Lambda|},\left[\tilde{Y}_{l^{\prime}}, Z\right]$ is still tangent to $M$.
} 
Proof. By Lemma D.1 it follows that

$$
\mu_{a}\left(g \partial_{p} f\right)=e^{-\mathcal{U}} \partial_{a}\left\{e^{\mathcal{U}} \mu_{a}(g p f)\right\}
$$

provided $g$ does not depend on $p$. Thus, since $u_{i, k}$ is locally bounded,

$$
\begin{aligned}
\alpha\left(a_{i}, a_{k}\right)= & \mu_{\underline{a}}\left(\nabla V\left(q_{i}-q_{k}\right)\left(\partial_{p_{i}} u_{i, k}-\partial_{p_{k}} u_{i, k}\right)\right) \\
= & e^{-\mathcal{U}} \partial_{a_{i}}\left\{e^{\mathcal{U}} \mu_{\underline{a}}\left(\nabla V\left(q_{i}-q_{k}\right) p_{i} u_{i, k}\right)\right\} \\
& -e^{-\mathcal{U}} \partial_{a_{k}}\left\{e^{\mathcal{U}} \mu_{\underline{a}}\left(\nabla V\left(q_{i}-q_{k}\right) p_{k} u_{i, k}\right)\right\} .
\end{aligned}
$$

To continue, notice that for each smooth $\varphi$,

$$
\begin{aligned}
\int e^{-\sum_{l} a_{l}} \prod_{l} \mathcal{Z}\left(a_{l}\right) \varphi(\underline{a}) \mu_{\underline{a}}\left(p_{i} \nabla V\left(q_{i}-q_{k}\right) u_{i, k}\right) \\
\quad=\mathbb{E}_{m_{0}}\left(\varphi\left(\underline{\mathcal{E}}^{0}\right) p_{i} \nabla V\left(q_{i}-q_{k}\right) u_{i, k}\right) \\
=-\mathbb{E}_{m_{0}}\left(\varphi\left(\underline{\mathcal{E}}^{0}\right) u_{i, k} L_{0}^{*} V\left(q_{i}-q_{k}\right)\right)+\mathbb{E}_{m_{0}}\left(\varphi\left(\underline{\mathcal{E}}^{0}\right) p_{k} \nabla V\left(q_{i}-q_{k}\right) u_{i, k}\right) \\
=-\mathbb{E}_{m_{0}}\left(\varphi\left(\underline{\mathcal{E}}^{0}\right) V\left(q_{i}-q_{k}\right) L_{0} u_{i, k}\right)+\mathbb{E}_{m_{0}}\left(\varphi\left(\underline{\mathcal{E}}^{0}\right) p_{k} \nabla V\left(q_{i}-q_{k}\right) u_{i, k}\right) \\
=-\mathbb{E}_{m_{0}}\left(\varphi\left(\underline{\mathcal{E}}^{0}\right) V\left(q_{i}-q_{k}\right) j_{i, k}\right)+\mathbb{E}_{m_{0}}\left(\varphi\left(\underline{\mathcal{E}}^{0}\right) p_{k} \nabla V\left(q_{i}-q_{k}\right) u_{i, k}\right) \\
=\int e^{-\sum_{l} a_{l}} \prod_{l} \mathcal{Z}\left(a_{l}\right) \varphi(\underline{a}) \mu_{\underline{a}}\left(p_{k} \nabla V\left(q_{i}-q_{k}\right) u_{i, k}\right),
\end{aligned}
$$

where we used the antisymmetry of $j_{i, k}$ with respect to $p$.

Hence

$$
\mu_{\underline{a}}\left(p_{i} \nabla V\left(q_{i}-q_{k}\right) u_{i, k}\right)=\mu_{\underline{a}}\left(p_{k} \nabla V\left(q_{i}-q_{k}\right) u_{i, k}\right)
$$

$m_{0}$-almost surely. Accordingly,

$$
\begin{aligned}
\alpha\left(a_{i}, a_{k}\right) & =\frac{1}{2} e^{-\mathcal{U}}\left(\partial_{a_{i}}-\partial_{a_{k}}\right)\left\{e^{\mathcal{U}} \mu_{\underline{a}}\left(\nabla V\left(q_{i}-q_{k}\right)\left(p_{i}+p_{k}\right) u_{i, k}\right)\right\} \\
& =e^{-\mathcal{U}}\left(\partial_{a_{i}}-\partial_{a_{k}}\right)\left\{e^{\mathcal{U}} \mu_{\underline{a}}\left(j_{i k} u_{i, k}\right)\right\} .
\end{aligned}
$$

The result follows since

$$
\begin{aligned}
\mu_{\underline{a}}\left(j_{i, k} u_{i, k}\right) & =\mu_{\underline{a}}\left(u_{i, k} L_{0} u_{i, k}\right)=\sigma^{2} \mu_{\underline{a}}\left(u_{i, k} S u_{i, k}\right) \\
& =-\sigma^{2} \sum_{j=i, k} \mu_{\underline{a}}\left(\left(X_{j} u_{i, k}\right)^{2}\right)=-\gamma^{2}\left(a_{i}, a_{k}\right) .
\end{aligned}
$$

We can rewrite (8.2) as

$$
\alpha\left(a_{i}, a_{k}\right)=\left(\partial_{a_{i}}-\partial_{a_{k}}\right) \gamma^{2}\left(a_{i}, a_{k}\right)+\left(\frac{\mathcal{Z}^{\prime}\left(a_{i}\right)}{\mathcal{Z}\left(a_{i}\right)}-\frac{\mathcal{Z}^{\prime}\left(a_{k}\right)}{\mathcal{Z}\left(a_{k}\right)}\right) \gamma^{2}\left(a_{i}, a_{k}\right) .
$$

Since $\frac{\mathcal{Z}^{\prime}(a)}{\mathcal{Z}(a)} \sim a^{-1}$, the regularity of $\alpha$ follows from that of $\gamma^{2}$ if we can prove that $\gamma^{2} \sim a_{1} a_{2}$.

Lemma 8.2. There exists $G \in \mathcal{C}^{\infty}\left([0, \infty)^{2}, \mathbb{R}\right)$ such that, for each $a_{1}, a_{2} \geq 0$,

$$
\gamma^{2}\left(a_{1}, a_{2}\right)=a_{1} a_{2} G\left(a_{1}, a_{2}\right) \geq 0,
$$

and furthermore $\alpha(0, a) \geq 0$ for all $a \geq 0$.

Proof. Observe that fixing the energy of the first particle $\mathcal{E}_{1}\left(q_{1}, p_{1}\right)=0$, it implies that $q_{1}=p_{1}=0$. So defining $\hat{u}(q, p)=u_{1,2}(0,0, q, p)$, it solves on $\mathbb{R}^{2}$ the equation $L \hat{u}=\hat{\boldsymbol{j}}$, where $L$ is the generator of the dynamics of a single isolated atom and $\hat{\boldsymbol{j}}=-\frac{1}{2} p \cdot \nabla V(q)=L^{*} V$. 
By the smoothness of $\gamma^{2}$ it follows that

$$
\gamma^{2}(0, a)=-\mu_{a}(\hat{\boldsymbol{j}} \hat{u})=\mu_{a}\left(L^{*} V \cdot \hat{u}\right)=\mu_{a}(V L \hat{u})=\mu_{a}(V \hat{\boldsymbol{j}})=0
$$

due to the symmetry of $\mu_{a}$ with respect to the transformation $p \rightarrow-p$. The structure of $\gamma^{2}$ then follows by the symmetry and smoothness of $\gamma^{2}$. The positivity follows by

$$
\gamma^{2}=-\mu_{a_{1}, a_{2}}\left(L_{0} u \cdot u\right)=\sigma^{2} \mu_{a_{1}, a_{2}}\left(\left(X_{1} u\right)^{2}+\left(X_{2} u\right)^{2}\right) .
$$

By (8.4) and (D.5),

$$
\alpha(a, 0)=2 a G(a, 0) \geq 0 .
$$

\section{Appendix A. Commutators}

This appendix collects some formulae concerning commutators for the unperturbed system $(\varepsilon=0)$. We use the notation of Proposition 6.1. In Section [5] we have already computed:

$$
\begin{gathered}
{\left[C_{0}, B\right]=\left(J p \cdot \partial_{q}+J \nabla U(q) \cdot \partial_{p}\right)=: C_{1}} \\
{\left[C_{1}, B\right]=4 \bar{U}^{\prime}(q) J q \cdot \partial_{q}-4\left\{\bar{U}^{\prime \prime}(q)|q|^{2}+\bar{U}^{\prime}\right\} J p \cdot \partial_{p}} \\
=: 2 C_{2}+\zeta(q) C_{0}=: 2 C_{2}+R_{2} .
\end{gathered}
$$

An explicit computation shows that

$$
C_{2}=\frac{p \cdot J \nabla U}{p^{2}} B+\frac{p \cdot \nabla U}{p^{2}} C_{1}-\frac{\|\nabla U\|^{2}}{p^{2}} C_{0} .
$$

The above formula shows that $C_{2}$, for small $p$ and large $q$, is not bounded by $B, C_{0}, C_{1}$ and, as we will see in the following, this forces us to compute

$$
\begin{aligned}
R_{3}=\left[C_{2}, B\right] & =-\left(J \nabla U(q) \cdot D^{2} U(q) \partial_{p}+J D^{2} U(q) p \cdot \partial_{q}\right) \\
& =-\left\{4 \bar{U}^{\prime \prime}\langle p, q\rangle J q+2 \bar{U}^{\prime} J p\right\} \partial_{q}-4\left(\bar{U}^{\prime}\right)^{2} J q \partial_{p} .
\end{aligned}
$$

By using the orthonormal base $Z_{0}, Z_{1}, Z_{2}$, defined in (5.5), we have that

$$
\begin{aligned}
R_{3}= & \sum_{j=0}^{2}\left\langle R_{3}, Z_{j}\right\rangle Z_{j} \\
= & \mathcal{N}^{-2}\left\langle R_{3}, B\right\rangle B+\mathcal{N}^{-2}\left\langle R_{3}, C_{1}\right\rangle C_{1}+\mathcal{N}^{-2}\left\langle R_{3},\left(C_{2}-C_{0}\right)\right\rangle\left(C_{2}-C_{0}\right) \\
= & 4 \mathcal{N}^{-2} \bar{U}^{\prime \prime}\langle q, p\rangle\langle J p, q\rangle B-\mathcal{N}^{-2}\left[4 \bar{U}^{\prime \prime}\langle q, p\rangle^{2}+2 \bar{U}^{\prime} p^{2}+8\left(\bar{U}^{\prime}\right)^{3} q^{2}\right] C_{1} \\
& -8 \mathcal{N}^{-2} \bar{U}^{\prime \prime} \bar{U}^{\prime}\langle q, p\rangle q^{2}\left(C_{2}-C_{0}\right),
\end{aligned}
$$

and an explicit calculation shows that

$$
\left\|R_{3}\right\|^{2}=\mathcal{N}^{-2}\left(\left|\left\langle R_{3}, B\right\rangle\right|^{2}+\left|\left\langle R_{3}, C_{1}\right\rangle\right|^{2}+\left|\left\langle R_{3},\left(C_{2}-C_{0}\right)\right\rangle\right|^{2}\right) \leq K .
$$

To conclude the first-order analysis we need to compute some more commutators:

$$
\begin{aligned}
& \left(\left[C_{0}, C_{1}\right]\right)=-B, \\
& \left(\left[C_{0}, C_{2}\right]\right)=0, \\
& \left(\left[C_{1}, C_{2}\right]\right)=: \rho C_{2}-\beta B,
\end{aligned}
$$

with $\rho$ and $\beta$ also bounded.

We also need some second-order commutators:

$$
\begin{aligned}
& {\left[B, C_{0}^{2}\right]=C_{0} B C_{0}-C_{0}^{2} B-C_{1} C_{0}=-2 C_{1} C_{0}+B,} \\
& {\left[C_{1}, C_{0}^{2}\right]=C_{0} B+B C_{0}=2 C_{0} B-C_{1} .}
\end{aligned}
$$




\section{Appendix B. Hypocoercivity estimates}

This appendix contains the core of the hypocoercivity argument. For our purposes it turns out to be more convenient to set it in an abstract setting.

Let $\mathcal{H}^{0}$ be a Hilbert space and $C_{0}, B$ be closed operators satisfying the relations A.1 A.6). Assume that $C_{k}, B, C_{k} C_{0}, B C_{0}$ all have a common core $D_{c}$. In addition, assume that for each $h, g \in D_{c}$ and $Z \in\left\{C_{0}, B\right\}$,

$$
\langle h, Z g\rangle=-\langle Z h, g\rangle
$$

where $\langle\cdot, \cdot\rangle$ is the scalar product of $\mathcal{H}^{0}$. Observe that the various constants that will appear in the results $(\tau, K$, etc.) do not depend on the scalar product $\langle h, g\rangle$.

We are interested in obtaining estimates in terms of the following generalized Sobolev norm:

$$
\|h\|_{\mathscr{H}^{1}}^{2}:=\|h\|^{2}+\sum_{k=0}^{2}\left\|C_{k} h\right\|^{2}+\|B h\|^{2} .
$$

To this end it turns out to be convenient to define the bilinear form:

$$
\begin{aligned}
\langle h, g\rangle\rangle:= & \langle h, g\rangle+\sum_{k=0}^{2} a_{k}\left\langle C_{k} h, C_{k} g\right\rangle+a_{3}\langle B h, B g\rangle \\
& -b_{0}\left\langle C_{0} h, C_{1} g\right\rangle-b_{0}\left\langle C_{1} h, C_{0} g\right\rangle-b_{1}\left\langle C_{1} h, C_{2} g\right\rangle-b_{1}\left\langle C_{2} h, C_{1} g\right\rangle,
\end{aligned}
$$

where $a_{k}>0, b_{k}>0$ will be chosen shortly (see (B.6) and (B.7)).

Lemma B.1. If for $\delta \in(0,1)$,

$$
a_{0} b_{1}^{2}+a_{2} b_{0}^{2} \leq a_{0} a_{1} a_{2}(1-\delta)^{2},
$$

then the quadratic form is positive definite and

$$
\langle h, h\rangle \geq\left(\|h\|^{2}+\delta \sum_{k=0}^{2} a_{k}\left\|C_{k} h\right\|^{2}+a_{3}\|B h\|^{2}\right)=\kappa\|h\|_{\mathscr{H}^{1}}^{2}
$$

with $\kappa=\min \left\{\delta a_{0}, \delta a_{1}, \delta a_{2}, a_{3}, 1\right\}$.

Proof. We have for any $\alpha_{0}, \alpha_{1}>0$,

$$
\begin{aligned}
\langle\langle h, h\rangle & -a_{3}\|B h\|^{2}-\|h\|^{2} \geq \sum_{k=0}^{2} a_{k}\left\|C_{k} h\right\|^{2}-\sum_{k=0}^{1}\left(b_{k} \alpha_{k}\left\|C_{k} h\right\|^{2}+b_{k} \alpha_{k}^{-1}\left\|C_{k+1} h\right\|^{2}\right) \\
= & \left(a_{0}-b_{0} \alpha_{0}\right)\left\|C_{0} h\right\|^{2}+\left(a_{1}-b_{0} \alpha_{0}^{-1}-b_{1} \alpha_{1}^{-1}\right)\left\|C_{1} h\right\|^{2}+\left(a_{2}-b_{1} \alpha_{1}\right)\left\|C_{2} h\right\|^{2} \\
= & \delta \sum_{k=0}^{2} a_{k}\left\|C_{k} h\right\|^{2}+\left(a_{0}(1-\delta)-b_{0} \alpha_{0}\right)\left\|C_{0} h\right\|^{2}+\left(a_{2}(1-\delta)-b_{1} \alpha_{1}\right)\left\|C_{2} h\right\|^{2} \\
& +\left(a_{1}(1-\delta)-b_{0} \alpha_{0}^{-1}-b_{1} \alpha_{1}^{-1}\right)\left\|C_{1} h\right\|^{2} .
\end{aligned}
$$

Then choosing

$$
\alpha_{0}=\frac{a_{0}(1-\delta)}{b_{0}}, \quad \alpha_{1}=\frac{a_{2}(1-\delta)}{b_{1}},
$$

the lemma follows immediately by condition (B.3).

On the other hand, the Schwarz inequality implies that there exists $K>0$ such that

$$
\langle h, h\rangle\rangle \leq K\left(\|h\|^{2}+\sum_{k=0}^{2}\left\|C_{k} h\right\|^{2}+\|B h\|^{2}\right) .
$$


Let $\mathcal{H}^{1}:=\left\{h \in \mathcal{H}^{0}:\langle\langle h, h\rangle\langle\infty\}\right.$. Clearly it is a Hilbert space, equivalent to $\mathscr{H}^{1}$, with scalar product $\langle\langle\cdot, \cdot\rangle\rangle$.

Lemma B.2. Given $C_{0}, B$ as described at the beginning of the section, there exists $\tau>0$ such that, for each $\sigma \in(0,1)$,

$$
\langle h, L h\rangle \leq-\tau \sigma^{2}\left\{\sum_{k=0}^{2}\left\|C_{k} h\right\|^{2}+\sum_{k=0}^{2}\left\|C_{k} C_{0} h\right\|^{2}+\|B h\|^{2}+\left\|B C_{0} h\right\|^{2}\right\} .
$$

Proof. This is a proof by boring computations. Let us start:

$$
\begin{aligned}
& \left\langle\langle h, L h\rangle=\sigma^{2}\left\langle h, C_{0}^{2} h\right\rangle+\left\{\sum_{k=0}^{2} a_{k}[\sigma^{2} \underbrace{\left\langle C_{k} C_{0}^{2} h, C_{k} h\right\rangle}_{\mathbf{I}_{\mathbf{A}, \mathbf{k}}}+\underbrace{\left\langle C_{k} B h, C_{k} h\right\rangle}_{\mathbf{I}_{\mathbf{B}, \mathbf{k}}}]\right.\right. \\
& +\sigma^{2} a_{3} \underbrace{\left\langle B C_{0}^{2} h, B h\right\rangle}_{\mathbf{I}_{\mathbf{B}, \mathbf{B}}}-\sum_{k=0}^{1} \sigma^{2} b_{k} \underbrace{\left(\left\langle C_{k} h, C_{k+1} C_{0}^{2} h\right\rangle+\left\langle C_{k} C_{0}^{2} h, C_{k+1} h\right\rangle\right)}_{\mathbf{I I}_{\mathbf{A}, \mathbf{k}}} \\
& -\sum_{k=0}^{1} b_{k} \underbrace{\left(\left\langle C_{k} h, C_{k+1} B h\right\rangle+\left\langle C_{k} B h, C_{k+1} h\right\rangle\right)}_{\mathbf{I I}_{\mathbf{B}, \mathbf{k}}}\} .
\end{aligned}
$$

Now we must look at all the terms one by one. We will use systematically (A.1)(A.5).

$$
\begin{aligned}
& \left\langle h, C_{0}^{2} h\right\rangle=-\left\langle C_{0} h, C_{0} h\right\rangle . \\
& \left(\mathbf{I}_{\mathbf{A}, \mathbf{0}}\right) \quad\left\langle C_{0}^{3} h, C_{0} h\right\rangle=-\left\langle C_{0}^{2} h, C_{0}^{2} h\right\rangle . \\
& \left(\mathbf{I}_{\mathbf{A}, \mathbf{1}}\right) \quad=-\left\langle C_{1} C_{0} h, C_{0} C_{1} h\right\rangle+\left\langle C_{0} B h, C_{1} h\right\rangle-\left\langle C_{1} h, C_{1} h\right\rangle \\
& =-\left\langle C_{1} C_{0} h, C_{1} C_{0} h\right\rangle+\left\langle C_{1} C_{0} h, B h\right\rangle-\left\langle B h, C_{0} C_{1} h\right\rangle \\
& -\left\langle C_{1} h, C_{1} h\right\rangle \\
& =-\left\langle C_{1} C_{0} h, C_{1} C_{0} h\right\rangle+\langle B h, B h\rangle-\left\langle C_{1} h, C_{1} h\right\rangle . \\
& \left(\mathbf{I}_{\mathbf{A}, \mathbf{2}}\right) \quad\left\langle C_{2} C_{0}^{2} h, C_{2} h\right\rangle=-\left\langle C_{2} C_{0} h, C_{2} C_{0} h\right\rangle . \\
& \left(\mathbf{I}_{\mathbf{B}, \mathbf{0}}\right) \quad\left\langle C_{0} B h, C_{0} h\right\rangle=\left\langle C_{1} h, C_{0} h\right\rangle . \\
& \left(\mathbf{I}_{\mathbf{B}, \mathbf{1}}\right) \quad\left\langle C_{1} B h, C_{1} h\right\rangle=2\left\langle C_{2} h, C_{1} h\right\rangle+\left\langle R_{2} h, C_{1} h\right\rangle . \\
& \left(\mathbf{I}_{\mathbf{B}, \mathbf{2}}\right) \quad\left\langle C_{2} B h, C_{2} h\right\rangle=\left\langle R_{3} h, C_{2} h\right\rangle . \\
& \left\langle B C_{0}^{2} h, B h\right\rangle=-\left\langle B C_{0} h, C_{0} B h\right\rangle-\left\langle C_{1} C_{0} h, B h\right\rangle \\
& \left(\mathbf{I}_{\mathbf{B}, \mathbf{B}}\right) \quad=-\left\langle B C_{0} h, B C_{0} h\right\rangle-\left\langle B C_{0} h, C_{1} h\right\rangle-\left\langle C_{1} C_{0} h, B h\right\rangle \\
& =-\left\langle B C_{0} h, B C_{0} h\right\rangle+\left\langle B h, C_{0} C_{1} h\right\rangle+\left\langle C_{1} h, C_{1} h\right\rangle-\left\langle C_{1} C_{0} h, B h\right\rangle \\
& =-\left\langle B C_{0} h, B C_{0} h\right\rangle-\langle B h, B h\rangle+\left\langle C_{1} h, C_{1} h\right\rangle \text {. }
\end{aligned}
$$


$\left(\mathbf{I I}_{\mathbf{A}, \mathbf{0}}\right) \quad\left\langle C_{0} C_{0}^{2} h, C_{1} h\right\rangle+\left\langle C_{0} h, C_{1} C_{0}^{2} h\right\rangle=-2\left\langle C_{0}^{2} h, C_{1} C_{0} h\right\rangle-\left\langle C_{0} h, C_{1} h\right\rangle$.

$\left(\mathbf{I I}_{\mathbf{A}, \mathbf{1}}\right) \quad\left\langle C_{1} C_{0}^{2} h, C_{2} h\right\rangle+\left\langle C_{1} h, C_{2} C_{0}^{2} h\right\rangle=-2\left\langle C_{1} C_{0} h, C_{2} C_{0} h\right\rangle-\left\langle C_{1} h, C_{2} h\right\rangle$.

$\left(\mathbf{I I}_{\mathbf{B}, \mathbf{0}}\right) \quad\left\langle C_{0} B h, C_{1} h\right\rangle+\left\langle C_{0} h, C_{1} B h\right\rangle=\left\langle C_{1} h, C_{1} h\right\rangle+2\left\langle C_{0} h, C_{2} h\right\rangle+\left\langle C_{0} h, R_{2} h\right\rangle$.

$\left(\mathbf{I I}_{\mathbf{B}, \mathbf{1}}\right) \quad\left\langle C_{1} B h, C_{2} h\right\rangle+\left\langle C_{1} h, C_{2} B h\right\rangle=2\left\langle C_{2} h, C_{2} h\right\rangle+\left\langle R_{2} h, C_{2} h\right\rangle+\left\langle C_{1} h, R_{3} h\right\rangle$.

Finally, we can put all the terms together, obtaining

$$
\begin{aligned}
\langle h, L h\rangle= & -\sigma^{2}\left\|C_{0} h\right\|^{2}-\left[b_{0}+\sigma^{2}\left(a_{1}-a_{3}\right)\right]\left\|C_{1} h\right\|^{2}-2 b_{1}\left\|C_{2} h\right\|^{2} \\
& -\sigma^{2}\left(a_{3}-a_{1}\right)\|B h\|^{2}+\left(a_{0}+\sigma^{2} b_{0}\right)\left\langle C_{0} h, C_{1} h\right\rangle+a_{1}\left\langle R_{2} h, C_{1} h\right\rangle \\
& -b_{0}\left\langle R_{2} h, C_{0} h\right\rangle-b_{1}\left\langle R_{2} h, C_{2} h\right\rangle+\left(2 a_{1}+\sigma^{2} b_{1}\right)\left\langle C_{1} h, C_{2} h\right\rangle \\
& -2 b_{0}\left\langle C_{0} h, C_{2} h\right\rangle+a_{2}\left\langle R_{3} h, C_{2} h\right\rangle-b_{1}\left\langle R_{3} h, C_{1} h\right\rangle \\
& -\underbrace{\sigma^{2}\left(\sum_{k=0}^{2} a_{k}\left\|C_{k} C_{0} h\right\|^{2}+a_{3}\left\|B C_{0} h\right\|^{2}-2 \sum_{k=0}^{1} b_{k}\left\langle C_{k} C_{0} h, C_{k+1} C_{0} h\right\rangle\right)}_{\text {III }} .
\end{aligned}
$$

By the same argument used in the proof of Lemma B.1, if for $0<\delta<1$ we have

$$
a_{0} b_{1}^{2}+a_{2} b_{0}^{2}<a_{0} a_{1} a_{2}(1-\delta)^{2},
$$

then III is bounded by $-\sigma^{2} \delta\left(\sum_{k} a_{k}\left\|C_{k} C_{0} h\right\|^{2}+a_{3}\left\|B C_{0} h\right\|^{2}\right)$.

Recalling (A.2) and (A.4), we have $\left\|R_{2} h\right\|^{2} \leq K_{2}\left\|C_{0} h\right\|^{2},\left\|R_{3} h\right\|^{2} \leq K_{3}\left\{\|B h\|^{2}+\right.$ $\left.\left\|C_{0} h\right\|^{2}+\left\|C_{1} h\right\|^{2}+\left\|C_{2} h\right\|^{2}\right\}$. Applying Schwarz, for each $\alpha_{1}, \alpha_{2}, \alpha_{3}, \alpha_{4}>0$, the first three lines of the above equation are bounded by

$$
\begin{aligned}
& -\left\{\sigma^{2}-\frac{1}{2} \alpha_{1}\left[a_{0}+\sigma^{2} b_{0}+K_{2} a_{1}\right]-K_{2}\left(b_{0}+b_{1}\right)-\alpha_{3} b_{0}-\frac{1}{2} K_{3} a_{2}-\frac{1}{2} b_{1} K_{3} \alpha_{4}\right\}\left\|C_{0} h\right\|^{2} \\
& -\left\{b_{0}-\sigma^{2}\left(a_{3}-a_{1}\right)-\frac{a_{0}+\sigma^{2} b_{0}+a_{1}}{2 \alpha_{1}}-\frac{2 a_{1}+\sigma^{2} b_{1}}{2 \alpha_{2}}-\frac{K_{3} a_{2}}{2}-\frac{\left(K_{3} \alpha_{4}^{2}+1\right) b_{1}}{2 \alpha_{4}}\right\}\left\|C_{1} h\right\|^{2} \\
& -\left\{2 b_{1}-\frac{1}{2} b_{1}-\frac{1}{2} \alpha_{2}\left(2 a_{1}+\sigma^{2} b_{1}\right)-\alpha_{3}^{-1} b_{0}-\frac{1}{2}\left(K_{3}+1\right) a_{2}-\frac{1}{2} b_{1} K_{3} \alpha_{4}\right\}\left\|C_{2} h\right\|^{2} \\
& -\left\{\sigma^{2}\left(a_{3}-a_{1}\right)-\frac{1}{2} K_{3} a_{2}-\frac{1}{2} K_{3} b_{1} \alpha_{4}\right\}\|B h\|^{2} .
\end{aligned}
$$

An inspection of the above expression shows that with the choices

$$
a_{0}=\sigma^{2} v^{7} ; a_{1}=\sigma^{2} v^{16} ; a_{2}=\sigma^{2} v^{18} ; a_{3}=v^{13} ; b_{0}=\sigma^{2} v^{12} ; b_{1}=\sigma^{2} v^{17}
$$

and

$$
\alpha_{1}=v^{-6} ; \alpha_{2}=v^{2} ; \alpha_{3}=v^{-5} ; \alpha_{4}=v,
$$

this implies that, by choosing $v$ small enough, there exists $\tau>0$ such that, for each $\sigma \in(0,1)$,

$$
\langle h, L h\rangle \leq-\tau \sigma^{2}\left\{\sum_{k=0}^{2}\left\|C_{k} h\right\|^{2}+\|B h\|^{2}+\sum_{k=0}^{2}\left\|C_{k} C_{0} h\right\|^{2}+\left\|B C_{0} h\right\|^{2}\right\} .
$$

Observe that (B.6) is satisfied by this choice. 


\section{Appendix C. A coordinate Change}

We study a change of coordinates in the case of one particle; for many particles one simply considers the product.

Let $S^{3}:=\left\{x \in \mathbb{R}^{4}:\|x\|=1\right\}$ and $M=\mathbb{R} \times S^{3} \subset \mathbb{R} \times \mathbb{R}^{2} \times \mathbb{R}^{2}, M_{+}=(0, \infty) \times S^{3} \subset M$. Let $\Psi: \mathbb{R}^{4} \backslash\{0\} \rightarrow M_{+} \subset \mathbb{R}^{5}$ be defined by

$$
\Psi(q, p)=\left(\sqrt{\frac{p^{2}}{2}+U(q)}, \frac{q \sqrt{U(q)}}{|q| \sqrt{\frac{p^{2}}{2}+U(q)}}, \frac{p}{\sqrt{p^{2}+2 U(q)}}\right)=:(r, \xi, \eta) .
$$

Remember that, by hypotheses, $U(q)=\bar{U}\left(q^{2}\right)$ and $\bar{U}$ is a strictly increasing function. We can then extend $\bar{U}$ to a smooth increasing function on $\mathbb{R}$ such that $\bar{U}(z) \geq 0$ if $z \geq 0$ It follows that $\rho(z):=\bar{U}^{-1}(z)$ is a well-defined smooth function on $\mathbb{R}$ such that $\rho(0)=0$.

One can readily check that the inverse $\Psi^{-1}: M_{+} \rightarrow \mathbb{R}^{4}$ is given by

$$
\Psi^{-1}(r, \xi, \eta)=\left(\sqrt{\rho\left(r^{2} \xi^{2}\right)} \frac{\xi}{\|\xi\|}, \sqrt{2} r \eta\right) .
$$

For the following it is convenient to introduce the function $\theta(z)=\sqrt{\rho(z) / z}, z \neq 0$. Notice that $\theta$ is smooth on $\mathbb{R}$ provided we set $\theta(0)=\sqrt{1 / \bar{U}^{\prime}(0)}$.

Next we transport the vector fields on $M$ by the usual formula $\Psi_{*} Z=(D \Psi Z)$ 。 $\Psi^{-1}$. The following lemma follows by the computation of $D \Psi$ that can be found in Appendix D.

Lemma C.1. With the above notation we have

$$
\begin{aligned}
& \tilde{C}_{0}:=\Psi_{\star} C_{0}=J \eta \cdot \partial_{\eta}, \\
& \tilde{B}:=\Psi_{*} B=\frac{\sqrt{2}}{\theta\left(r^{2} \xi^{2}\right)}\left\{\eta-\xi \frac{\langle\xi, \eta\rangle}{\xi^{2}}\left[1-\bar{U}^{\prime}\left(\rho\left(r^{2} \xi^{2}\right)\right) \theta\left(r^{2} \xi^{2}\right)^{2}\right]\right\} \partial_{\xi} \\
& -\sqrt{2} \bar{U}^{\prime}\left(\rho\left(r^{2} \xi^{2}\right)\right) \theta\left(r^{2} \xi^{2}\right) \xi \partial_{\eta}, \\
& \tilde{Y}:=\Psi_{\star} Y=\partial_{r} .
\end{aligned}
$$

Define the function

$$
\Gamma(z):=1-\bar{U}^{\prime}(\rho(z)) \theta(z)^{2}
$$

and notice that it is continuous in 0 and $\Gamma(0)=0$. It is then natural to write

$$
\begin{aligned}
& \tilde{B}=\frac{\sqrt{2}}{\theta\left(r^{2} \xi^{2}\right)}\left\{\tilde{B}^{0}+\Gamma\left(r^{2} \xi^{2}\right) \tilde{B}^{1}\right\}, \\
& \tilde{B}^{0}=\eta \partial_{\xi}-\xi \partial_{\eta}, \quad \tilde{B}^{1}=\xi \partial_{\eta}-\frac{\langle\xi, \eta\rangle}{\xi^{2}} \xi \partial_{\xi} .
\end{aligned}
$$

Notice that $\theta\left(r^{2} \xi^{2}\right)$ and $\Gamma\left(r^{2} \xi^{2}\right)$ are smooth functions of $\xi$ and $r$; consequently $\tilde{B}$ is a smooth vector field. In addition, both $\tilde{B}^{0}$ and $\tilde{B}^{1}$ are tangent to the surfaces $\{r\} \times S^{3} \subset M$.

Remark C.2. The vector fields $\tilde{C}_{0}, \tilde{B}, \tilde{Y}$ are defined only on $M_{+}$but admit a smooth canonical extension to all $M$. We will use the same notation to designate such an extension.

\footnotetext{
${ }^{5}$ Clearly the extension is arbitrary, but this is irrelevant in the following.
} 
It follows that there exist smooth vector fields $\tilde{C}_{1}, \tilde{C}_{2}$ on $M$ such that $\tilde{C}_{i}=\Psi_{*} C_{i}$ on $M_{+}$.

\section{Appendix D. Microcanonical measure}

We collect here some properties about the microcanonical measure $\mu_{a}$ (and its product version $\mu_{\underline{a}}$ ).

Let us first recall the definition and some formulas. Microcanonical measure is defined as the conditional measure on the energy shell $\Sigma_{a}=\left\{(q, p) \in \mathbb{R}^{4}:\|p\|^{2} / 2+\right.$ $U(q)=a\}$. This means that for every continuous function $\phi: \mathbb{R}_{+} \rightarrow \mathbb{R}$ and $f$ on $\mathbb{R}^{4}$ we have

$$
\int \phi\left(\mathcal{E}_{0}(p, q)\right) f(p, q) d m_{0}=\int_{\mathbb{R}_{+}} \phi(a) \mu_{a}(f) e^{-a} \mathcal{Z}(a) d a
$$

Standard formulas give that

$$
\mathcal{Z}(a)=\int_{\Sigma_{a}}\left(p^{2}+U^{\prime}(q)^{2}\right)^{-1 / 2} d \sigma_{\Sigma_{a}}(p, q)
$$

and

$$
\mu_{a}(f)=\mathcal{Z}(a)^{-1} \int_{\Sigma_{a}} f(p, q)\left(p^{2}+U^{\prime}(q)^{2}\right)^{-1 / 2} d \sigma_{\Sigma_{a}}(p, q)
$$

where $\sigma_{\Sigma_{a}}$ is the Lebesgue measure on the energy shell.

We need an integration by parts formula for the microcanonical measure $\mu_{a}$. This is provided by the following proposition.

Proposition D.1. For all $f, g$ continuous locally bounded functions on $\mathbb{R}^{4}$, such that $g$ does not depend on $p_{k}\left(\partial_{p_{k}} g \equiv 0\right)$, and $f$ is differentiable in $p_{k}$, then

$$
\mu_{\underline{a}}\left(g \partial_{p_{k}} f\right)=\left(\partial_{a_{k}} \mu_{\underline{a}}\right)\left(g p_{k} f\right)+\frac{\mathcal{Z}^{\prime}\left(a_{k}\right)}{\mathcal{Z}\left(a_{k}\right)} \mu_{\underline{a}}\left(g p_{k} f\right), \quad m_{0, \beta}-a . s ., a_{k}>0 .
$$

Proof. Because of the product structure of $\mu_{\underline{a}}$, we can just consider only one site and we drop the index $k$ of it.

Let us recall that from the definition of $\mu_{a}$ it follows that, for all smooth bounded functions $f, g$ and $\varphi: \mathbb{R}_{+} \rightarrow \mathbb{R}$ supported away from zero,

$$
\int_{\mathbb{R}^{4}} d p d q e^{-H_{0}(p, q)} \varphi\left(H_{0}\right) f(p, q)=\int_{\mathbb{R}_{+}} d a e^{-a} \varphi(a) \mathcal{Z}(a) \mu_{a}(f) .
$$

Thus

$$
\begin{array}{r}
\int_{\mathbb{R}_{+}} d a e^{-a} \varphi(a) \mathcal{Z}(a) \mu_{a}\left(g \partial_{p} f\right)=\int_{\mathbb{R}^{4}} d p d q e^{-H_{0}} \varphi\left(H_{0}\right) g(q) \partial_{p} f(p, q) \\
=\int_{\mathbb{R}^{4}} d p d q e^{-H_{0}}\left\{-\varphi^{\prime}\left(H_{0}\right)+\varphi\left(H_{0}\right)\right\} g(q) p f(p, q) \\
=\int_{\mathbb{R}_{+}} d a e^{-a}\left\{-\varphi^{\prime}(a)+\varphi(a)\right\} \mathcal{Z}(a) \mu_{a}(g p f) \\
=\int_{\mathbb{R}_{+}} d a e^{-a} \varphi(a) \partial_{a}\left(\mathcal{Z}(a) \mu_{a}(g p f)\right) \\
=\int_{\mathbb{R}_{+}} d a e^{-a} \varphi(a) \mathcal{Z}(a) \partial_{a}\left(\mu_{a}(g p f)\right)+\int_{\mathbb{R}_{+}} d a e^{-a} \varphi(a) \frac{\mathcal{Z}^{\prime}(a)}{\mathcal{Z}(a)} \mathcal{Z}(a) \mu_{a}(g p f) .
\end{array}
$$

The relation (D.4) follows for any bounded smooth $f, g$. The result follows by approximations. 
Formula (D.3) is difficult to be used directly, but exploiting the symmetry and the convexity of the potential, it is possible to write this microcanonical expectation as as integral on the 3 -dimensional sphere of radius 1 with respect to the corresponding uniform measure. In fact the strict convexity of $U$ makes the energy shell very close to a sphere for small energy $a$, and $\mu_{a}$ close to the uniform measure on this sphere. We want to study this more precisely.

Recall the change of coordinates $\Psi$ introduced in Section 7 , by (7.5) and its inverse (C.1). Recall also the notation $\theta(z)=\sqrt{\rho(z) / z}$, and that $\theta(0) \rightarrow \sqrt{1 / \bar{U}^{\prime}(0)}$.

\section{Lemma D.2.}

$$
\mathcal{Z}(a)=4 \omega_{4} a \int_{S^{3}}\left[\bar{U}^{\prime}\left(\rho\left(a \xi^{2}\right)\right)\right]^{-1} d \sigma(\xi, \eta),
$$

where we have used polar coordinates in four dimensions, $\sigma$ is the uniform probability measure on $S^{3}$, the unit four-dimensional ball, and $\omega_{4}$ is its volume. Furthermore,

$$
\mu_{a}(f)=\frac{4 \omega_{4} a}{\mathcal{Z}(a)} \int_{S^{3}} f \circ \Psi^{-1}(a, \xi, \eta)\left[\bar{U}^{\prime}\left(\rho\left(\sqrt{a} \xi^{2}\right)\right)\right]^{-1} d \sigma(\xi, \eta) .
$$

Proof. Instead of computing with differential forms it turns out to be more efficient to use the following trick.

Consider the change of variables $\tilde{\Psi}:\left(\mathbb{R}^{4} \backslash\{0\}\right) \times \mathbb{R}_{+} \rightarrow \mathbb{R}^{5}$ defined by

$$
\tilde{\Psi}(q, p, s)=\left(\frac{p^{2}}{2}+U(q), \frac{s q \sqrt{U(q)}}{|q| \sqrt{\frac{p^{2}}{2}+U(q)}}, \frac{s p}{\sqrt{p^{2}+2 U(q)}}\right) .
$$

Note that $\tilde{\Psi}$ is invertible and, setting $\varrho(\tilde{\xi}, \tilde{\eta})=\sqrt{\tilde{\xi}^{2}+\tilde{\eta}^{2}}$,

$$
\tilde{\Psi}^{-1}(a, \tilde{\xi}, \tilde{\eta})=\left(\sqrt{\rho\left(a \varrho^{-2} \tilde{\xi}^{2}\right)} \frac{\tilde{\xi}}{\|\tilde{\xi}\|}, \varrho^{-1} \sqrt{2 a} \tilde{\eta}, \varrho\right) .
$$

Then, given any two compact support functions $g \in \mathcal{C}^{0}\left(\mathbb{R}_{+}, \mathbb{R}\right), f \in \mathcal{C}^{0}\left(\mathbb{R}^{4}, \mathbb{R}\right)$, we can write

$$
\begin{aligned}
\int_{\mathbb{R}^{5}} g(s) f(p, q) d q d p d s=\int_{\mathbb{R}^{5}}\left[g f|\operatorname{det}(D \tilde{\Psi})|^{-1}\right] \circ \tilde{\Psi}^{-1}(a, \tilde{\xi}, \tilde{\eta}) d a d \tilde{\xi} d \tilde{\eta} \\
\quad=4 \omega_{4} \int_{\mathbb{R}^{2} \times S^{3}} g(s)\left[f|\operatorname{det}(D \tilde{\Psi})|^{-1}\right] \circ \tilde{\Psi}^{-1}(a, s \xi, s \eta) s^{3} d a d s d \sigma(\xi, \eta) .
\end{aligned}
$$

In Lemma D.3 we compute the determinant of

$$
D \tilde{\Psi} \circ \tilde{\Psi}^{-1}(a, \tilde{\xi}, \tilde{\eta})=\left(\begin{array}{lll}
\frac{\partial a}{\partial q} & \frac{\partial a}{\partial p} & \frac{\partial a}{\partial s} \\
\frac{\partial \tilde{\xi}}{\partial q} & \frac{\partial \tilde{\xi}}{\partial p} & \frac{\partial \tilde{\xi}}{\partial p} \\
\frac{\partial \tilde{\eta}}{\partial q} & \frac{\partial \tilde{\eta}}{\partial p} & \frac{\partial \tilde{\eta}}{\partial p}
\end{array}\right) \circ \tilde{\Psi}^{-1}(a, \tilde{\xi}, \tilde{\eta})
$$

for $\tilde{\xi}^{2}+\tilde{\eta}^{2}=1$, obtaining

$$
\operatorname{det}\left(D \tilde{\Psi} \circ \tilde{\Psi}^{-1}\right)=\frac{\tilde{U}^{\prime}\left(\rho\left(a \xi^{2}\right)\right)}{a}
$$

Thus, if we take a sequence of $g_{n}$ that converges weakly to the delta function on one, we have the formula

$$
\int_{\mathbb{R}^{4}} f(p, q) d q d p=4 \omega_{4} \int_{M_{+}} f \circ \tilde{\Psi}^{-1}(a, \xi, \eta) \frac{a}{\tilde{U}^{\prime}\left(\rho\left(a \xi^{2}\right)\right)} d a d \sigma(\xi, \eta) .
$$


Accordingly, for each $g \in L^{1}\left(\mathbb{R}_{+}\right)$we can write

$$
\int_{\mathbb{R}^{4}} f(p, q) g\left(\mathcal{E}^{0}\right) d q d p=4 \omega_{4} \int_{M_{+}} g(a) f \circ \tilde{\Psi}^{-1}(a, \xi, \eta) \frac{a}{\tilde{U}^{\prime}\left(\rho\left(a \xi^{2}\right)\right)} d a d \sigma(\xi, \eta) .
$$

On the other hand by the definition of the microcanonical measure $\mu_{a}$ :

$$
\int_{\mathbb{R}^{4}} f(p, q) g\left(\mathcal{E}^{0}\right) d q d p=\int_{\mathbb{R}_{+}} g(a) \mathcal{Z}(a) \mu_{a}(f) d a .
$$

The above, by the arbitrariness of $g$, yields the following formula for the microcanonical expectation:

$$
\mu_{a}(f)=4 \omega_{4} a \mathcal{Z}(a)^{-1} \int_{S^{3}} f \circ \tilde{\Psi}^{-1}(a, \xi, \eta)\left[\tilde{U}^{\prime}\left(\rho\left(a \xi^{2}\right)\right)\right]^{-1} d \sigma(\xi, \eta) .
$$

Putting $f=1$ in (D.10) implies the formula for $\mathcal{Z}(a)$.

Lemma D.3. Proof of equation (D.9).

Proof. An explicit computation of the derivative yields

$$
D \tilde{\Psi} \circ \tilde{\Psi}^{-1}=\left(\begin{array}{ccc}
2 \tilde{U}^{\prime}\left(\rho\left(a \xi^{2} \varrho^{-2}\right)\right) \theta\left(a \xi^{2} \varrho^{-2}\right) \sqrt{a} \varrho^{-1} \xi & \varrho^{-1} \sqrt{2 a} \eta & 0 \\
\frac{\varrho}{\sqrt{a} \theta}\left[\mathbb{1}-\frac{\xi \otimes \xi}{\xi^{2}}\left\{1-\eta^{2} \varrho^{-2} \tilde{U}^{\prime}(\rho) \theta^{2}\right]\right. & -\frac{1}{\sqrt{2 a} \varrho} \xi \otimes \eta & \varrho^{-1} \xi^{t} \\
-\frac{1}{\sqrt{a} \varrho} \theta \tilde{U}^{\prime}(\rho) \eta \otimes \xi & \frac{\varrho}{\sqrt{2 a}}\left[\mathbb{1}-\varrho^{-2} \eta \otimes \eta\right] & \varrho^{-1} \eta^{t}
\end{array}\right) .
$$

We want to compute the determinant for $(\xi, \eta) \in S^{3}$, i.e. $\varrho=1$. If we multiply the first row by $(2 a)^{-1} \xi_{1}$ and we sum it to the second, then by $(2 a)^{-1} \xi_{2}$ and sum it to the third, by $(2 a)^{-1} \eta_{1}$ and sum to the fourth and finally by $(2 a)^{-1} \eta_{2}$ and sum it to the last row, we have

$$
\begin{aligned}
& \operatorname{det}\left(D \tilde{\Psi} \circ \tilde{\Psi}^{-1}\right)=\operatorname{det}\left(\begin{array}{ccc}
2 \tilde{U}^{\prime}\left(\rho\left(a \xi^{2}\right)\right) \theta\left(a \xi^{2}\right) \sqrt{a} \xi & \sqrt{2 a} \eta & 0 \\
\frac{1}{\sqrt{a} \theta}\left[\mathbb{1}-\frac{\xi \otimes \xi}{\xi^{2}}\left\{1-\tilde{U}^{\prime}(\rho) \theta^{2}\right]\right. & 0 & \xi^{t} \\
0 & \mathbb{1}(2 a)^{-\frac{1}{2}} & \eta^{t}
\end{array}\right) \\
& =\operatorname{det}\left(\begin{array}{ccc}
2 \tilde{U}^{\prime}\left(\rho\left(a \xi^{2}\right)\right) \theta\left(a \xi^{2}\right) \sqrt{a} \xi & 0 & -2 a \eta^{2} \\
\frac{1}{\sqrt{a} \theta}\left[\mathbb{1}-\frac{\xi \otimes \xi}{\xi^{2}}\left\{1-\tilde{U}^{\prime}(\rho) \theta^{2}\right]\right. & 0 & \xi^{t} \\
0 & \mathbb{1}(2 a)^{-\frac{1}{2}} & \eta^{t}
\end{array}\right) \\
& =\frac{1}{2 a} \operatorname{det}\left(\begin{array}{cc}
2 \tilde{U}^{\prime}\left(\rho\left(a \xi^{2}\right)\right) \theta\left(a \xi^{2}\right) \sqrt{a} \xi & -2 a \eta^{2} \\
\frac{1}{\sqrt{a} \theta}\left[\mathbb{1}-\frac{\xi \otimes \xi}{\xi^{2}}\left\{1-\tilde{U}^{\prime}(\rho) \theta^{2}\right]\right. & \xi^{t}
\end{array}\right) \\
& =\frac{1}{2 a} \operatorname{det}\left(\begin{array}{cc}
0 & -2 a \eta^{2} \\
\frac{1}{\sqrt{a} \theta}\left[\mathbb{1}-\frac{\xi \otimes \xi}{\xi^{2}}\left\{1-\eta^{-2} \tilde{U}^{\prime}(\rho) \theta^{2}\right]\right. & \xi^{t}
\end{array}\right) .
\end{aligned}
$$


From the above, the lemma follows 6

Corollary D.4. Let $f$ be a continuous function of $(q, p)$. Then the following formula holds:

$$
\mu_{a}(f(q, p))=\int_{S^{3}} f \circ \Psi^{-1}(a, \xi, \eta) d \sigma(\xi, \eta)+\mathcal{O}(a),
$$

where $\sigma$ is the uniform probability measure on $S^{3}$, and $\mathcal{O}(a)$ is a smooth function of order a as $a \rightarrow 0$.

Notice that because of (D.10), since $0<c \leq \tilde{U}^{\prime} \leq c^{-1}<+\infty$, the microcanonical measure is uniformly equivalent to the uniform measure on the unit sphere, and, for any $a>0$, and any positive $f$ :

$$
c^{-2} \int_{S^{3}} f \circ \tilde{\Psi}^{-1}(a, \xi, \eta) d \sigma(\xi, \eta) \leq \mu_{a}(f) \leq c^{2} \int_{S^{3}} f \circ \tilde{\Psi}^{-1}(a, \xi, \eta) d \sigma(\xi, \eta) .
$$

\section{Appendix E. The Gaussian case}

Just to give a concrete idea of what we are doing and to provide some concrete intuition, here we discuss the case in which we have just two particles and both $U$ and $V$ are quadratic, the point being that such a case can be solved explicitly and hence provides a guidance for what can be expected in the general case.

Let us consider a Hamiltonian system with four degrees of freedom $\left(q_{1}, p_{1}, q_{2}, p_{2}\right)$ $\in \mathbb{R}^{8}$ given by

$$
H_{\varepsilon}:=\frac{1}{2}\left\{\left\|p_{1}\right\|^{2}+\left\|p_{2}\right\|^{2}\right\}+\frac{1}{2}\left\{\left\|q_{1}\right\|^{2}+\left\|q_{2}\right\|^{2}\right\}+\varepsilon\left\|q_{1}-q_{2}\right\|^{2}
$$

plus random forces that conserve the kinetic energy (that is, independent diffusions on the circles $\left\|p_{i}\right\|^{2}=$ const $)$. To this end consider the vector fields

$$
X_{i}:=p_{i, 1} \partial_{p_{i, 2}}-p_{i, 2} \partial_{p_{i, 1}} .
$$

The generator is thus given by

$$
L_{\varepsilon}:=\left\{H_{\varepsilon}, \cdot\right\}+\sigma^{2} \sum_{i=1}^{2} X_{i}^{2}=A_{\varepsilon}+\sigma^{2} S .
$$

The energies of the two particles are $\mathcal{E}_{i}=\frac{1}{2}\left\|p_{i}\right\|^{2}+\frac{1}{2}\left\|q_{i}\right\|^{2}+\frac{\varepsilon}{2}\left\|q_{1}-q_{2}\right\|^{2}$, and

$$
\begin{aligned}
& \partial_{t} \mathcal{E}_{1}=\varepsilon\left\langle q_{2}-q_{1}, p_{1}+p_{2}\right\rangle=: \varepsilon j, \\
& \partial_{t} \mathcal{E}_{2}=-\varepsilon j
\end{aligned}
$$

give the current. A direct computation shows that, setting

$$
u:=\frac{1}{2}\left\{\left\|q_{2}\right\|^{2}-\left\|q_{1}\right\|^{2}\right\}-\sigma^{-2} q_{2} \cdot p_{1}+\sigma^{-2} q_{1} \cdot p_{2},
$$

the following holds true:

$$
L_{\varepsilon} u=j+\varepsilon \sigma^{-2}\left\{\left\|q_{1}\right\|^{2}-\left\|q_{2}\right\|^{2}\right\}=: \tilde{j} .
$$

\footnotetext{
${ }^{6}$ Just remember that $\operatorname{det}(\mathbb{1}-b v \otimes v)=1-b v^{2}$ since $v$ and any vector perpendicular to $v$ are eigenvectors with eigenvalues $1-b v^{2}$ and 1 respectively.
} 
Accordingly, if we rescale the time by $\varepsilon^{-2}$ and we look at the random variables $\mathcal{E}_{i, \varepsilon}(t):=\mathcal{E}_{i}\left(\varepsilon^{-2} t\right)$, then

$$
\begin{array}{r}
\mathcal{E}_{1, \varepsilon}(t)-\mathcal{E}_{1, \varepsilon}(0)=\varepsilon \int_{0}^{\varepsilon^{-2} t}\left(L_{\varepsilon} u\right)(s) d s-\varepsilon^{2} \sigma^{-2} \int_{0}^{\varepsilon^{-2} t}\left(\left\|q_{1}(s)\right\|^{2}-\left\|q_{2}(s)\right\|^{2}\right) d s \\
=\varepsilon\left(u\left(\varepsilon^{-2} t\right)-u(0)\right)+\varepsilon M_{\varepsilon^{-2} t}^{u}-\sigma^{-2} \int_{0}^{t}\left(\mathcal{E}_{1, \varepsilon}(\tau)-\mathcal{E}_{2, \varepsilon}(\tau)\right) d \tau \\
-\varepsilon^{2} \sigma^{-2} \int_{0}^{\varepsilon^{-2} t}\left[\left(\left\|q_{1}(s)\right\|^{2}-\left\|p_{1}(s)\right\|^{2}\right)-\left(\left\|q_{2}(s)\right\|^{2}-\left\|p_{2}(s)\right\|^{2}\right)\right] d s+\mathcal{O}\left(\varepsilon^{3}\right),
\end{array}
$$

where the martingale $M_{t}^{u}$ has quadratic variation given by

$$
\left\langle M^{u}\right\rangle_{t}=\sigma^{2} \int_{0}^{t}\left[\left(X_{1} u(s)\right)^{2}+\left(X_{2} u(s)\right)^{2}\right] d s .
$$

We first show that the average of the last term on the RHS of (E.2) tends to 0. Observe that

$$
\begin{aligned}
\left(\left\|q_{1}\right\|^{2}-\left\|p_{1}\right\|^{2}\right) & -\left(\left\|q_{2}\right\|^{2}-\left\|p_{2}\right\|^{2}\right) \\
& =-L_{\varepsilon}\left(p_{1} \cdot q_{1}-p_{2} \cdot q_{2}+\frac{1}{2}\left(\left\|q_{1}\right\|^{2}-\left\|q_{2}\right\|^{2}\right)\right)-\varepsilon\left(\left\|q_{1}\right\|^{2}-\left\|q_{2}\right\|^{2}\right) .
\end{aligned}
$$

Letting $v=p_{1} \cdot q_{1}-p_{2} \cdot q_{2}+\frac{1}{2}\left(\left\|q_{1}\right\|^{2}-\left\|q_{2}\right\|^{2}\right)$, the last term on the RHS of (E.2) can be rewritten as

$$
\varepsilon^{2}\left(v\left(\varepsilon^{-2} t\right)-v(0)\right)+\varepsilon^{2} M_{\varepsilon^{-2} t}^{v}+\varepsilon^{3} \int_{0}^{\varepsilon^{-2} t}\left(\left\|q_{1}(s)\right\|^{2}-\left\|q_{2}(s)\right\|^{2}\right) d s .
$$

It is easy to show that the average goes to 0 as $\varepsilon \rightarrow 0$.

It remains to compute the limit of the martingale $\varepsilon M_{\varepsilon^{-2} t}^{u}$. To this purpose one has to compute the limit of its quadratic variation:

$$
\left\langle\varepsilon M^{u}\right\rangle_{\varepsilon^{-2} t}=\varepsilon^{2} \sigma^{-2} \int_{0}^{\varepsilon^{-2} t}\left[\left(q_{2,1} p_{1,2}-q_{2,2} p_{1,1}\right)^{2}+\left(q_{1,2} p_{2,1}-q_{1,1} q_{2,2}\right)^{2}\right] d s .
$$

After some explicit calculation, one can find 4th-order polynomials $\psi, \phi, \chi$ such that

$$
\left(q_{2,1} p_{1,2}-q_{2,2} p_{1,1}\right)^{2}+\left(q_{1,2} p_{2,1}-q_{1,1} q_{2,2}\right)^{2}=2 \mathcal{E}_{1} \mathcal{E}_{2}+L_{\varepsilon} \psi+S \phi+\varepsilon \chi .
$$

The term $L_{\varepsilon} \psi+\varepsilon \chi$ does not contribute. For the term $S \phi$, by using the Schwarz inequality,

$$
\begin{aligned}
-\int_{0}^{t} d \tau \int S \phi F_{\tau} d \mu_{\varepsilon}=\int_{0}^{t} d \tau \sum_{i=1,2} \int\left(X_{i} \phi\right)\left(X_{i} F_{\tau}\right) d \mu_{\varepsilon} \\
\leq\left(\int_{0}^{t} \mathcal{D}\left(F_{\tau}\right) d \tau\right)^{1 / 2}\left(\int_{0}^{t} \sum_{i=1,2} \int\left(X_{i} \phi\right)^{2} F_{\tau} d \mu_{\varepsilon} d \tau\right)^{1 / 2} \\
\leq\left(\int_{0}^{t} \mathcal{D}\left(F_{\tau}\right) d \tau\right)^{1 / 2}\left(\int_{0}^{t} \sum_{i=1,2}\|F\|_{L}^{2}\left[\int\left(X_{i} \phi\right)^{4} d \mu_{\varepsilon}\right]^{\frac{1}{2}} d \tau\right)^{1 / 2} \leq C \sqrt{t} .
\end{aligned}
$$

We have obtained that $\mathcal{E}_{1, \varepsilon}, \mathcal{E}_{2, \varepsilon}$ converge to the (degenerate) diffusion on $R_{+}^{2}$ generated by

$$
\mathcal{L}=\sigma^{-2} \mathcal{E}_{1}^{-1} \mathcal{E}_{2}^{-1}\left(\partial_{\mathcal{E}_{1}}-\partial_{\mathcal{E}_{2}}\right) \mathcal{E}_{1}^{2} \mathcal{E}_{2}^{2}\left(\partial_{\mathcal{E}_{1}}-\partial_{\mathcal{E}_{2}}\right)
$$


corresponding to the stochastic differential equation:

$$
d \mathcal{E}_{1}=\sigma^{-1} \sqrt{2 \mathcal{E}_{1} \mathcal{E}_{2}} d w_{t}-2 \sigma^{-2}\left(\mathcal{E}_{1}-\mathcal{E}_{2}\right) d t=-d \mathcal{E}_{2}
$$

with $w_{t}$ the standard Wiener process.

\section{ACKNOWLEDGMENTS}

It is a pleasure to thank Clement Mouhot for many very useful discussions. The authors are also indebted to Boguslaw Zegarliński for helpful suggestions.

\section{REFERENCES}

[1] C. Bernardin, S. Olla, Fourier's law for a microscopic model of heat conduction, Journal of Statistical Physics, 118 (2005), nos. 3/4, 271-289. MR2185330 (2006j:82058)

[2] F. Bonetto, J.L. Lebowitz, L. Rey-Bellet, Fourier's law: A challenge to theorists, Mathematical Physics 2000, Imperial College Press, London, 2000, pp.128-150. MR 1773043 (2001g:82067)

[3] S. Cerrai, Ph. Clément, Well-posedness of the martingale problem for some degenerate diffusion processes occurring in dynamics of populations, Bull. Sci. Math. 128 (2004) 355-389. MR2066345 (2005h:60249)

[4] Dmitry Dolgopyat, Carlangelo Liverani, Energy transfer in a fast-slow Hamiltonian system, Communications in Mathematical Physics, 308 (2011), N. 1, 201-225.

[5] M. I. Freidlin, Fluctuations in dynamical systems with averaging (Russian)Dokl. Akad. Nauk SSSR 226 (1976), no. 2, 273-276. MR0410796 (53:14539)

[6] M.I. Freidlin, A.D. Wentzell, Random Perturbations of Dynamical Systems, 2nd edn., Springer, Heidelberg, 1998. MR1652127 (99h:60128)

[7] L. Hormander, Hypoelliptic second order differential equations. Acta Math. 119 (1967), 147171. MR0222474 (36:5526)

[8] Y. Kifer, Some recent advances in averaging. In: Modern Dynamical Systems and Applications, pp. 385-403. Cambridge University Press, Cambridge, 2004. MR2093312 (2005h:37067)

[9] T. Komorowski, C. Landim, S. Olla, Fluctuations in Markov Processes, Springer, to appear (2012).

[10] S. Olla, M. Sasada, Macroscopic energy diffusion for a chain of anharmonic oscillators, arXiv 2011 vol. math-ph, http://arxiv.org/abs/1109.5297v1.

[11] S. Olla, S. Varadhan, H. Yau, Hydrodynamical limit for a Hamiltonian system with weak noise, Commun. Math. Phys. 155 (1993), 523-560. MR1231642 (94k:60158)

[12] Z. Rieder, J. L. Lebowitz, and E. Lieb, Properties of a Harmonic Crystal in a Stationary Nonequilibrium State, J. Math. Phys. 8 (1967), 1073.

[13] S. Sethuraman, S.R.S. Varadhan, H.T. Yau, Diffusive limit of a tagged particle in asymmetric simple exclusion processes, Comm. Pure Appl. Math. 53 (2000), no. 8, 972-1006. MR 1755948 (2001k:60146)

[14] S.R.S. Varadhan, Nonlinear diffusion limit for a system with nearest neighbor interactions. II, Asymptotic problems in probability theory: stochastic models and diffusions on fractals (Sanda/Kyoto, 1990), 75-128, Pitman Res. Notes Math. Ser., 283, Longman Sci. Tech., Harlow, 1993. MR 1354152 (97a:60144)

[15] Cedric Villani, Hypocoercivity. Mem. Amer. Math. Soc. 202 (2009), no. 950, 141 pp. MR2562709(2011e:35381)

Dipartimento di Matematica, II Università di Roma (Tor Vergata), Via della Ricerca SCientifica, 00133 Roma, Italy

E-mail address: liverani@mat.uniroma2.it

CEREMADE, UMR CNRS 7534, Université PARis-Dauphine, 75775 Paris-Cedex 16, France and inRIA - Université Paris Est, Cermics, Projet MiCMaC, Ecole des Ponts Paristech, 6 \& 8 Av. Pascal, 77455 Marne-la-Vallée Cedex 2, France

E-mail address: olla@ceremade.dauphine.fr 